\title{
Laser-induced fluorescence measurement of very slow neutral flows in a dusty plasma experiment $\oplus^{\circledR}$
}

Cite as: Rev. Sci. Instrum. 91, 063504 (2020); https://doi.org/10.1063/5.0006684

Submitted: 05 March 2020 . Accepted: 22 May 2020 . Published Online: 09 June 2020

R. S. Marshall (iD), and P. M. Bellan (i)

COLLECTIONS

EP This paper was selected as an Editor's Pick

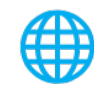

\begin{tabular}{|c|c|c|c|}
\hline & $\begin{array}{c}\text { Nanopositioning } \\
\text { Systems }\end{array}$ & $\begin{array}{c}\text { Modular } \\
\text { Motion Control }\end{array}$ & $\begin{array}{c}\text { AFM and NSOM } \\
\text { Instruments }\end{array}$ \\
\hline MAD CITY LABS INC. \\
www.madcitylabs.com
\end{tabular}




\title{
Laser-induced fluorescence measurement of very slow neutral flows in a dusty plasma experiment 라
}

\author{
Cite as: Rev. Sci. Instrum. 91, 063504 (2020); doi: 10.1063/5.0006684 \\ Submitted: 5 March 2020 - Accepted: 22 May 2020 • \\ Published Online: 9 June 2020
}

R. S. Marshalla) (D) and P. M. Bellan (iD

AFFILIATIONS
Applied Physics and Materials Science, California Institute of Technology, Pasadena, California 91125, USA

a) Author to whom correspondence should be addressed: rmarshal@caltech.edu

\begin{abstract}
Laser-Induced Fluorescence (LIF) provides the temperature and flow velocity of a target species by direct measurement of its velocity distribution via Doppler shift. A LIF diagnostic has been developed at the Caltech water-ice dusty plasma experiment that uses an ultranarrow tunable diode laser to pump the $\lambda_{v a c}=696.735 \mathrm{~nm}$ argon neutral transition. A photomultiplier detects fluorescence emission at $\lambda_{v a c}$ $=772.633 \mathrm{~nm}$. Signal to noise ratios in excess of 100 are achieved along with a high degree of reproducibility between measurements. A Labview program fully automates data collection throughout the three-dimensional plasma volume by controlling four stepper motors and recording measured data. The argon neutral temperature is measured to be slightly above room temperature. Challenges such as the lack of absolute calibration of diode lasers and wavelength drift due to slight changes in ambient room conditions are overcome to measure bulk neutral flow speeds on the order of 1-2 m/s with resolution on the order of $2 / 3$ of a meter per second. High-speed video shows that introducing an argon flow to a cloud of ice grains causes the cloud of ice grains to move and change shape. Ice grain motion is analyzed and found to be in agreement with neutral LIF flow measurements. Surprisingly, when the flow ceases, the ice grain cloud reverts to its original location and shape.
\end{abstract}

Published under license by AIP Publishing. https://doi.org/10.1063/5.0006684

\section{INTRODUCTION}

Laser-Induced Fluorescence (LIF) is a plasma spectroscopic diagnostic that can be used to measure the velocity distribution of a target species and so give the species temperature and flow speed. LIF works by exciting an atom or an ion from some initial state to an excited state and then measuring the fluorescence photons emitted as the atom decays out of the excited state to a third, different state. LIF was first performed in the 1970s using tunable dye lasers. ${ }^{1,2}$ Performance and convenience were improved when Severn et al. replaced the dye laser with a tunable diode laser, thereby demonstrating Tunable Diode-Laser Induced Fluorescence (TD-LIF) on the $668.429 \mathrm{~nm}$ argon II ion line. ${ }^{3}$

The temperature of argon neutral gas in both the dust-free and dusty plasma environments has recently been measured on the ground-based PK3 experiment in Germany using absorption spectroscopy, a method similar to LIF. ${ }^{4}$ One shortcoming associated with absorption spectroscopy is that it is a line-averaged measurement.
The addition of a photomultiplier tube (PMT) perpendicular to the excitation beam in an absorption spectroscopy experiment that detects fluorescence photons turns it into an LIF experiment. This extra hardware gives LIF a measurement localized in three dimensions. The first two dimensions of localization come from the cross section of the beam itself (as in absorption spectroscopy) and the third dimension of localization comes from the location along the beam path intersected by the PMT line of sight.

Over the past few decades, LIF has been used in a myriad of different ways. LIF is an established method to measure xenon neutral and ion exit speeds from Hall thrusters. ${ }^{5,6}$ By splitting one beam into two and using independent chopping, LIF has been used to simultaneously measure two components of the ion velocity distribution function where the components are of the order of $\sim 100$ 's of $\mathrm{m} / \mathrm{s}$. Because of the localization of the beam and detector, LIF is capable of measuring density profiles in three dimensions. ${ }^{8}$ PLIF (Planar LIF) imaging using a camera sensor to detect LIF emission from a planar excitation beam has been used to build an image of density. 
The shift between the double-peaked LIF spectrum from Zeeman splitting can even be used to measure magnetic fields. ${ }^{10}$

In this manuscript, we present TD-LIF measurements of the temperature and flow speed of neutral argon atoms in both dustfree and astrophysically relevant dusty environments through the introduction of frozen water-ice dust grains. Information on the astrophysical relevance and how the dust grains grow is given in Refs. 11 and 12. We report results using the argon neutral LIF scheme that pumps on the $\lambda_{v a c}=696.735 \mathrm{~nm}$ transition between the metastable $\left({ }^{2} P_{3 / 2}^{0}\right) 4 \mathrm{~S}$ level and the excited $\left({ }^{2} P_{1 / 2}^{0}\right) 4 \mathrm{P}$ level. Decay from the $\left({ }^{2} P_{1 / 2}^{0}\right) 4 \mathrm{P}$ excited level to the $\left({ }^{2} P_{1 / 2}^{0}\right) 4 \mathrm{~S}$ level produces $\lambda_{v a c}$ $=772.633 \mathrm{~nm}$ fluorescence photons that the PMT detects. ${ }^{13,14}$ Key results are as follows: (i) LIF works not only on a dust-free plasma but also on an astrophysically relevant dusty plasma, (ii) exploitation of symmetry allows measurement of the in-plane two dimensional flow velocity vector as a function of position $\{x, y, z\}$ in a threedimensional plasma volume, and (iii) flow speeds are measured and resolved with the sub-linewidth resolution.

This paper is organized as follows: Section II describes the Caltech water-ice dusty plasma experiment and the LIF diagnostic. Section III discusses the physical origin of LIF and how the temperature and flow speed are deduced from measurements. Section IV presents the results and discussion. Section $V$ presents the conclusion.

\section{EXPERIMENTAL SETUP}

The Caltech water-ice dusty plasma experiment is similar to an earlier experiment at the Max Planck Institute ${ }^{15}$ but has two important differences: (i) The electrode separation is adjustable and (ii) water molecules come from the water vapor pressure associated with a canister of liquid water rather than from creation of water in situ. Basic details about the experiment can be found in Ref. 16, with the main difference here being that the aluminum electrodes have been replaced by copper electrodes. Copper is more thermally conductive, which allows for more efficient cooling of the electrodes.

The experiment is housed in a $15.24 \mathrm{~cm}$ spherical-square vacuum chamber. A capacitively coupled RF discharge plasma is created between a pair of $6 \mathrm{~cm}$ diameter parallel plate copper electrodes separated by an adjustable gap. The electrodes are electrically driven by a custom-made power supply that generates $1-3 \mathrm{~W}$ of 13.56 $\mathrm{MHz}$ RF. The electrodes have cold fingers that pass through the vacuum chamber flanges to thermally connect to liquid nitrogen $\left(\mathrm{LN}_{2}\right)$ Dewars.

Figure 1 shows front view (a) and top view (b) schematic diagrams of the Caltech water-ice dusty plasma experiment as it has been setup to perform TD-LIF. A Toptica DLC Pro controller and a Toptica DL Pro ultra-narrow tunable diode laser-head are used. The DL Pro laser-head is capable of producing up to $\sim 35 \mathrm{~mW}$ of continuous beam power with a $\sim 150 \mathrm{kHz}$ linewidth. The beam traverses a beam-splitter and a mechanical chopper that chops the two resulting beams at $1 \mathrm{kHz}$. Both beams are coupled to optical fibers with collimators that direct the beams into the plasma. The collimation is slightly less than perfect with an opening angle $\theta \approx 0.33^{\circ}$. Over the distances traversed in the dusty plasma experiment, beam divergence is assumed to be negligibly small. A moveable barrier between the chopper wheel and the optical fiber entrances allows only one beam to pass through the plasma at a time. The key feature is that the fibers send the two laser beams into the plasma from opposite sides so that light from the " $+z$ beam" is traveling along the same path but in the direction opposite of the light from the " $-z$ beam." The $+z$ and $-z$ beams are named for the beam directions. Light from the $+z$ beam propagates in the positive $z$ direction from small $z$ to large $z$ and light from the $-z$ beam propagates from large $z$ to small $z$. A PMT utilizes a $1 \mathrm{~nm}$ full width half max line filter so that it only detects $772.633 \mathrm{~nm}$ fluorescence photons. It views the beams at a $90^{\circ}$ angle. The PMT signal goes to a lock-in amplifier, which is synchronized to the chopper.

In Fig. 1(b), the dashed black line and thick black dots on the optical fibers and PMT indicate that the $-z$ beam, the $+z$ beam, and the PMT are all mounted on the same rigid structure. This rigid structure is mounted on three stepper motors, which allow the two beams and the PMT to move together in three dimensions. The $1 \mathrm{D}$ translating barrier, also shown in Fig. 1(b), is moved by a fourth stepper motor. With these four motional degrees of freedom, the TD-LIF diagnostic is completely automated in Labview and can scan the plasma over a three-dimensional volume, with either the $+z$ or the $-z$ beam illuminating the plasma.

The three-dimensional perspective in Fig. 2 shows the relative orientation of the gas nozzles and the laser beams. Figures 3(a) and

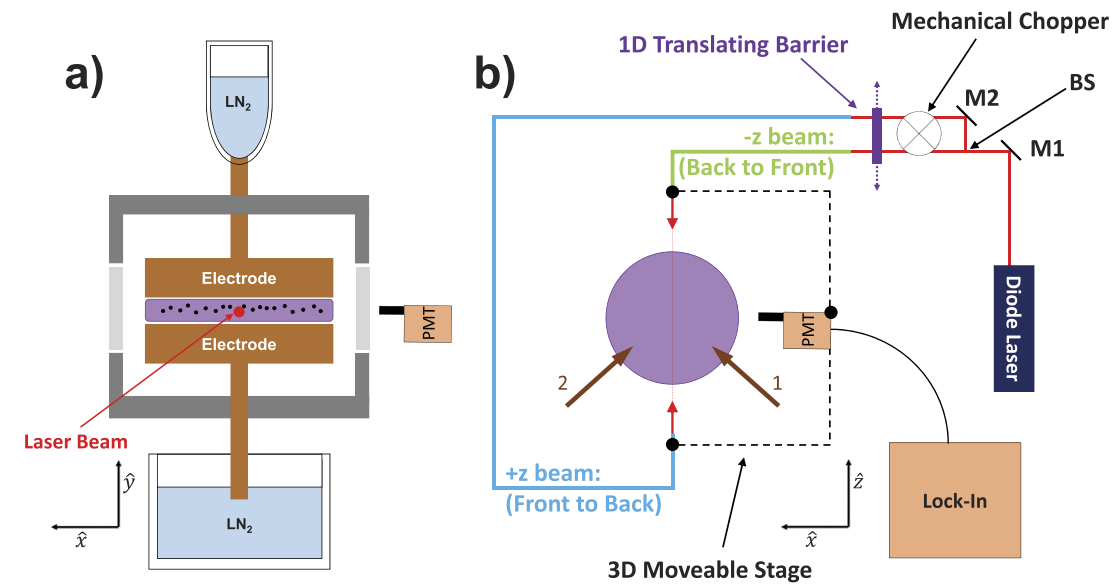

FIG. 1. Schematic representations of the LIF experiment on the Caltech waterice dusty plasma. (a) is the front view and $(b)$ is the top view. The bold green and blue lines represent the optical fibers that direct the light to the plasma. The rigid structure that moves the fibers and PMT in unison is shown by the three black dots connected by the dashed lines. Brown arrows 1 and 2 oriented $45^{\circ}$ inwards represent the nozzles through which argon gas enters the chamber. $\mathrm{M} 1$ and M2 are mirrors and BS is a beam-splitter. Coordinate directions are denoted by the set of axes on each sub-figure. 


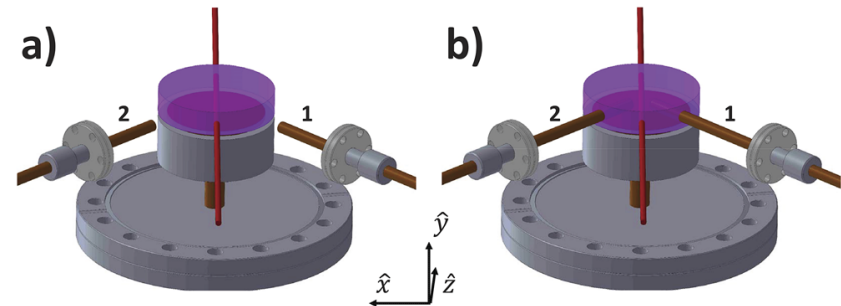

FIG. 2. Sketch showing the bottom electrode and 6 " Conflat flange with the plasma (purple) in the middle. Top electrode is not shown because it obstructs the view. The laser beam is the red line passing through in the $\hat{z}$ direction. The numbered copper-colored tubes are the gas nozzles. (a) and (b) are the same, the only difference being that the copper gas nozzles are outside the plasma or inside the plasma, respectively.

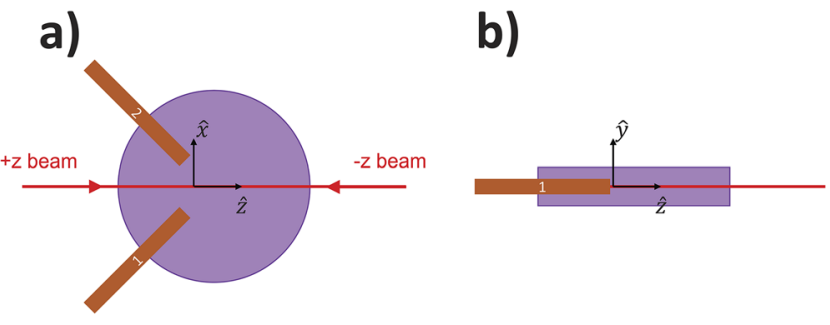

FIG. 3. Sketch to show the origin of the stepper motor coordinate system. (a) is the top view looking in $-\hat{y}$ and $(b)$ is a side view looking in $+\hat{x}$.

3 (b) show the coordinate system, where $x=0$ is defined to be midway between both nozzles, $y=0$ is defined to be in the middle of each nozzle, and $z=0$ is defined to be the edge of the nozzles. This origin $(0,0,0)$ will be used when moving the stepper motors to collect data from the plasma in a three-dimensional volume.

This motorized system can be programmed to measure LIF at any series of $(x, y, z)$ positions in the plasma. The biggest limitation is the finite cross-sectional area of the $-z$ and $+z$ beams. This poses little challenge when measuring LIF throughout the bulk volume of the plasma but becomes critical for measuring on length scales smaller than the diameter of the beams, for example, in the plasma sheath regions. Measuring detail in the sheath would require a smaller diameter laser beam. Additionally, it is observed when measuring LIF in the sheath of the plasma, that LIF signal amplitude quickly diminishes becoming too small to detect. Thus, results in this paper are limited to the bulk of the plasma.

The imposed symmetry between the gas inlet nozzles and the laser beam path shown in Figs. 2 and 3 will later be exploited to determine the $v_{x}$ and $v_{z}$ components of the in-plane flow velocity using only a single laser beam.

\section{LIF THEORY}

Because of the discrete nature of atomic line transitions, LIF can be used to measure the temperature and flow speed of any specific target species in the plasma as long as an appropriate laser and a defined LIF scheme both exist. In order to explain how to measure target species temperature and bulk flow speed, first, we will derive the mathematical form that the LIF signal should take.

As mentioned in the Introduction, the neutral argon LIF scheme used pumps an argon neutral metastable state with $\lambda_{v a c}$ $=696.735 \mathrm{~nm}$ and observes emission at $\lambda_{v a c}=772.633 \mathrm{~nm}$. While a stationary neutral atom will absorb radiation with wavelength $\lambda_{v a c}$ $=696.735 \mathrm{~nm}$, atoms in a plasma are moving and the light that each atom sees will be Doppler shifted based on the component of its velocity in the direction of the laser beam.

The Doppler shifted angular frequency is given by

$$
\omega=\omega_{0}-k_{0} v_{z}
$$

where $\omega_{0}=2 \pi f_{0}$ is the angular frequency of the unshifted source light, $k_{0}=2 \pi / \lambda_{0}$ is the wavenumber of the unshifted source light, and $v_{z}$ is the speed of the atom projected in the direction of the laser beam.

Rearranging Eq. (1) gives

$$
v_{z}=c \frac{\lambda-\lambda_{0}}{\lambda},
$$

where $v_{z}$ is the speed of the atom projected in the direction of the laser beam, $c$ is the speed of light, $\lambda_{0}$ is the wavelength of the unshifted light source, and $\lambda$ is the wavelength of light seen by the atom.

The Maxwell-Boltzmann distribution for atoms moving in the $\hat{z}$ direction with no mean bulk flow speed is

$$
f\left(v_{z}\right)=\sqrt{\frac{m}{2 \pi \kappa T}} e^{-\frac{m v_{z}^{2}}{2 \kappa T}} .
$$

Substituting $v_{z}$ in Eq. (3) with the Doppler shift relationship in Eq. (2) yields the mathematical form of the LIF signal,

$$
f(\lambda)=\sqrt{\frac{m}{2 \pi \kappa T}} e^{-\frac{\left(\lambda-\lambda_{0}\right)^{2}}{2\left(\sqrt{\frac{\kappa T}{m}} \frac{\lambda}{c}\right)^{2}}} .
$$

The measured LIF signal is fit to the Gaussian shape in Eq. (4). The temperature is determined by the width of the best-fit Gaussian and the bulk flow speed is determined using its center position $\lambda_{0}$.

\section{A. Temperature}

The temperature of the target species is simply the random motion of the species. This random motion corresponds to the width of the Gaussian in Eq. (4). Solving for the full width half max (FWHM) of the spectrum in Eq. (4) gives

$$
\mathrm{FWHM}=2 \lambda \sqrt{\frac{2 \kappa T}{m c^{2}} \log (2)}=7.7 \times 10^{-5} \lambda \sqrt{\frac{T}{m}},
$$

where $T$ has units of $\mathrm{eV}$ and $m$ has units of amu.

\section{B. Flow speed}

The flow speed of the target species is the bulk motion of the species. Bulk motion shifts the center position $\lambda_{0}$ of the Gaussian in Eq. (4). For the neutral argon species, there would be no bulk flow detected if the best-fit Gaussian has $\lambda_{0}=\lambda_{v a c}=696.735 \mathrm{~nm}$. However, for $\lambda_{0} \neq 696.735 \mathrm{~nm}$, the center has been shifted due to a bulk flow. In this case, the bulk flow speed is then determined using Eq. (2) to be $v_{z}=\Delta f \lambda_{0}=c\left(\frac{\lambda_{\text {vac }}-\lambda_{0}}{\lambda_{\text {vac }}}\right)$. 


\section{RESULTS AND DISCUSSION}

The diagnostic records the LIF signal amplitude from the lockin amplifier as a function of tunable diode laser piezo voltage. Changing the electric potential on the laser piezo alters the wavelength of light emitted by the laser. In order to convert from diode laser piezo voltage to wavelength, the user must know the exact wavelength at a single voltage (typically found using a reference) and then use the factory calibration factor $(21 \mathrm{GHz}=39.2 \mathrm{~V}$ change in the potential applied to the laser piezo tuning actuator) to find the wavelength everywhere else. The LIF signal as a function of wavelength is then fit to the Gaussian in Eq. (4) to provide temperature or flow speed, as shown in Secs. III A or III B. The TD-LIF diagnostic developed at Caltech produces very reproducible results with signal to noise ratios routinely in excess of 100 .

The Toptica DL Pro laser head has a $\sim 150 \mathrm{kHz}$ linewidth while the natural linewidth of the neutral argon $\lambda_{v a c}=696.735 \mathrm{~nm}$ absorption line is $\Delta f=5.6 \mathrm{MHz}{ }^{10}$ The Doppler width for thermal argon neutrals at $300 \mathrm{~K}$ is calculated to be $\sim 1 \mathrm{GHz}$, so the diode laser emits light as an effective $\delta$-function compared to other line profiles.

\section{A. Temperature measurements}

Doppler widths measured by the LIF diagnostic are on the order of $1 \mathrm{GHz}$. It was surprising to see that a diode laser illuminating the plasma with only a few milliwatts of pump beam was strong enough to enter a power-broadened regime and give a spurious power-dependent temperature reading. ${ }^{17}$ Optical density filters are used to reduce the beam power to the order of $300 \mu \mathrm{W}$, the point at which the measured LIF temperature no longer depends on the laser beam power and is considered accurate.

\section{Cooling electrodes}

With no $\mathrm{LN}_{2}$ cooling, the neutral argon temperature is typically measured to be $350 \mathrm{~K}$ compared with the $\sim 295 \mathrm{~K}$ room temperature. Similar results were found on the PK4 experimental setup at Baylor University where an early version of the diagnostic was installed and operated for two weeks. Upon cooling the electrodes with $\mathrm{LN}_{2}$, the LIF diagnostic indicates that the neutral argon temperature is much lower. Figure 4 shows the measured neutral argon temperature as a function of time while the electrodes are cooling. It takes about $30 \mathrm{~min}$ for the electrodes to cool from room temperature to $\sim 150 \mathrm{~K}$, and it similarly takes about $30 \mathrm{~min}$ for the argon neutrals to cool from $\sim 350 \mathrm{~K}$ to $\sim 200 \mathrm{~K}$.

\section{Demonstration that LIF works with ice grains}

One of the most important results is that the LIF diagnostic still works in a water-ice dusty environment. Figure 5 shows a measurement taken with water-ice dust present. A temperature of $192 \mathrm{~K}$ was determined.

\section{B. Flow measurements}

While measuring neutral argon temperature via LIF proved straight-forward after accounting for power broadening, measurement of flow speed was anything but straight-forward. Prior to the development of the LIF diagnostic, there had not been measurements of neutral flow velocity profiles inside a dusty plasma experiment. Many experiments, such as PK4 on the International Space Station, use precise electronic flow controllers to carefully regulate

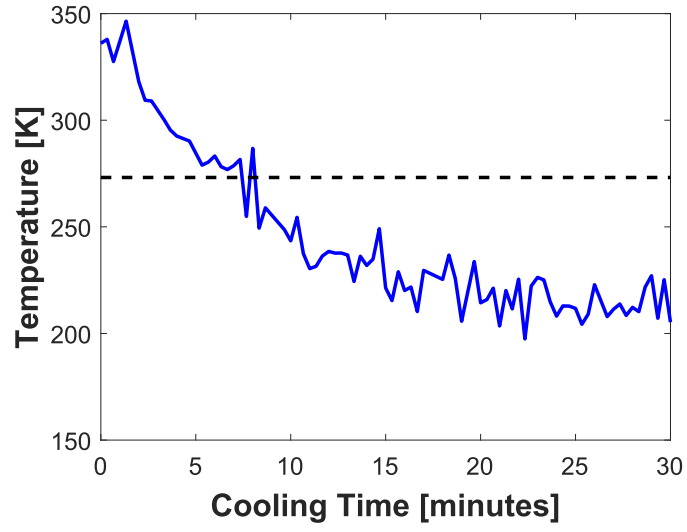

FIG. 4. Temperature of neutral argon atoms measured by LIF as a function of the time that the electrode cold fingers have been exposed to $L_{2}$. Over the $\sim 30$ min that the electrodes cool, the measured temperature of the neutral argon drops The black dashed line represents water freezing at $273 \mathrm{~K}$.

the flow entering the vacuum chamber where plasma is created. ${ }^{18}$ These flow controllers allow the flow to be known at the inlet into the chamber; however, the velocity profile after the flow leaves the nozzle and interacts with the existing plasma is still unknown. An LIF diagnostic can be used to quantify the unknown velocity profile inside the plasma. The Caltech experiment lacks an electronic flow controller. Instead, a mechanical leak valve is used. A consequence of this is that very little is known about how much gas passes through the leak valve control and how fast the gas is traveling. For reference, $\kappa T=m_{\text {argon }} v_{t h}^{2} / 2$ gives the room temperature thermal velocity of argon to be $v_{t h} \approx 300 \mathrm{~m} / \mathrm{s}$.

When measuring the temperature, it does not matter whether the gas nozzle configuration is as in Fig. 2(a) where the nozzles are outside of the plasma and on the order of $5 \mathrm{~cm}$ away

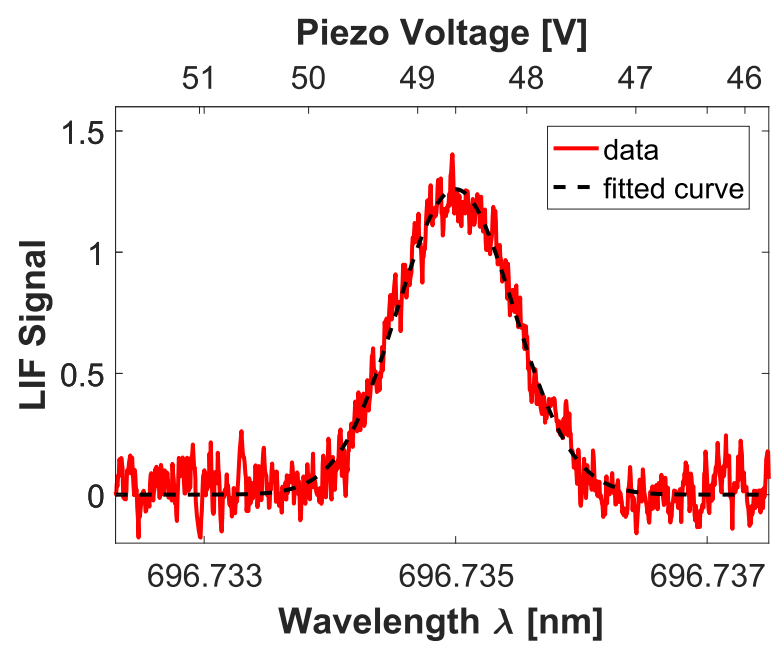

FIG. 5. LIF signal from neutral argon species in astrophysically relevant cold water-ice dusty plasma. 
from the counter-propagating laser beams or if the configuration is as in Fig. 2(b) where the nozzles are immediately adjacent to the counter-propagating beams. Initial attempts to measure flow speed were made with the nozzles in the configuration of Fig. 2(a). In this configuration, measurement of a bulk flow proved unsuccessful.

The key parameter needed to quantify bulk flow speed is the shift of the measured Gaussian from its Doppler-free value $\lambda_{v a c}$. This is challenging because diode lasers do not have an absolutely calibrated wavelength. Converting from the laser piezo voltage (frequency shift) to an absolute wavelength requires more information than the diode laser can provide. Consequently, it is impossible to determine the bulk flow speed simply by shining a laser beam through a plasma and then measuring a fluorescence signal, whereas this works for determining the temperature.

Several different methods were attempted before a successful flow speed measurement was achieved. One unsuccessful method was to obtain an absolute calibration between the piezo voltage and wavelength using the absorption spectrum from a separate plasma in a small fluorescent lamp starter as an absolute reference. ${ }^{19}$ This requires using a beam splitter to perform LIF on the Caltech plasma and absorption spectroscopy on the lamp starter plasma simultaneously. Since the lamp starter is small and sealed, it is assumed to have no flow, and thus, the peak absorption would define $v=0$. This absorption reference scheme established that the flow speed in the Caltech plasma was very small because the two spectra were perfectly superimposed within the available resolution. It was not possible to make a credible measurement of the flow speed. Absolute calibration was also attempted using the Lamb Dip, which has been successfully used to measure slow flows in plasma. ${ }^{20}$ However, the Lamb Dip was relatively small and requires operation at a pressure much lower than the pressure of interest.

In addition to the difficulty of the flow velocity being very small, the measurement is challenging because the diode laser wavelength constantly drifts due to slight changes in ambient conditions. Thus, any absolute calibration becomes inaccurate over time and a constant absolute calibration is required. While the diode laser has an ultra-narrow linewidth, in $20 \mathrm{~min}$, the wavelength typically drifts $\sim 10 \mathrm{MHz}$ to $20 \mathrm{MHz}$ and, over the course of $3 \mathrm{~h}$, by over $250 \mathrm{MHz}$. This drift would be negligible for measuring $v_{\text {flow }} \gg 200 \mathrm{~m} / \mathrm{s}$ flows but makes it impossible to measure $v_{\text {flow }} \leq 5 \mathrm{~m} / \mathrm{s}$. A $5 \mathrm{~m} / \mathrm{s}$ flow corresponds to a frequency shift of $14.36 \mathrm{MHz}$ between the two counterpropagating Gaussian peaks. This shift is so small that it would be masked by the laser drift.

Locking the laser wavelength to an external device seems like a logical approach to counteract the laser drifting. Unfortunately, the laser cannot be locked while scanning. An attempt was made to use the built-in PID locking capability of the DLC Pro to lock the laser wavelength using the absorption spectroscopy signal from the fluorescent lamp starter. Unfortunately, the lamp starter proved to be an unreliable reference because the plasma inside the lamp starter is not stable and its light intensity changes in the order of $10 \mathrm{~s}$ of seconds to minutes. We also tried to lock the laser to a specific wavelength using a wavemeter as a feedback source and then to average over a long time to obtain the LIF signal as a function of wavelength directly. This also failed because the dusty plasma light intensity changes sufficiently in a few minutes to make these data meaningless. It was determined that satisfactory results could not come from a single long-averaging method of data collection. Instead, a different technique using many 5-10 s measurements taken in succession and analyzed together was found to work and will be presented in Sec. IV B 1.

The final key to successfully measuring flow was to move the gas inlet nozzles from their original positions outside the plasma as in Fig. 2(a) to a position inside the plasma much closer to the pumping laser beam as in Fig. 2(b).

\section{Single position measurement}

A counter-propagating beam approach was developed to measure the extremely slow flows. This technique involves repeating the measurement 15 times for a total of 30 datasets at a given position. Figure 6 shows typical data taken with this approach. This method can be considered to be, in effect, a double modulation scheme where the first level of modulation is from the chopper and the second level of modulation is the alternation between the counter-propagating beams.

The diode laser is set to scan a range of $\Delta \lambda=7 \mathrm{pm}$ around $\lambda_{v a c}$ over 5 seconds. Specifically, the laser scans from $\lambda=696.7385 \mathrm{~nm}$ to $\lambda=696.7315 \mathrm{~nm}$ and back to $\lambda=696.7385 \mathrm{~nm}$ in $5 \mathrm{~s}$. The lockin amplifier averages with a time constant $\tau=10 \mathrm{~ms}$. The steps $i$ taken to measure flow and how long they take to complete $\Delta t_{i}$ are as follows:

1. The translating barrier moves to block the $+z$ beam and to allow the $-z$ beam to pass. No measurement is made while the barrier is moving $\left(\Delta t_{1}=10 \mathrm{~s}\right)$.

2. The LIF measurement is saved and plotted in red in Fig. 6(a). The measurement is fit to a Gaussian and the center piezo voltage of the Gaussian is plotted in red in Fig. 6(b) $\left(\Delta t_{2}=5 \mathrm{~s}\right)$.

3. The translating barrier moves to block the $-z$ beam and to allow the $+z$ beam to pass. No measurement is made while the barrier is moving $\left(\Delta t_{3}=10 \mathrm{~s}\right)$.

4. The LIF measurement is saved and plotted in blue in Fig. 6(a). The measurement is fit to a Gaussian and the center piezo voltage of the Gaussian is plotted in blue in Fig. 6(b) $\left(\Delta t_{4}\right.$ $=5 \mathrm{~s}$ ).

5. Steps 1-4 above are repeated 14 more times. The total time to complete 30 scans is $T=15\left(\Delta t_{1}+\Delta t_{2}+\Delta t_{3}+\Delta t_{4}\right) \approx 450 \mathrm{~s}$.

Figure 6(a) shows all 30 individual sets of data taken during the $\sim 450$ s procedure superimposed: the 15 red traces represent measurements from the $-z$ beam and the 15 blue traces represent measurements from the $+z$ beam. Figure 6 (a) shows that the results are extremely reproducible. Figure 6(b) shows the center piezo voltages for the 30 Gaussians in Fig. 6(a). The scan number ranges from 1 to 30 because 30 datasets were taken. The $-z$ beam (red) was taken first, so there are red data points for odd numbered scans and blue data points for the even numbered scans taken with the $+z$ beam. The black dashed lines alternating between the red and blue circles represent the order the data were taken starting with the bottom red data point on the left, then the blue data point with the dashed line connecting, and so on.

The 30 LIF measurements plotted in Fig. 6(a) are deliberately shown as a function of piezo voltage, not wavelength. There are three reasons for this. (i) The wavelength as a function of piezo voltage differs slightly for each scan because the laser is drifting; 

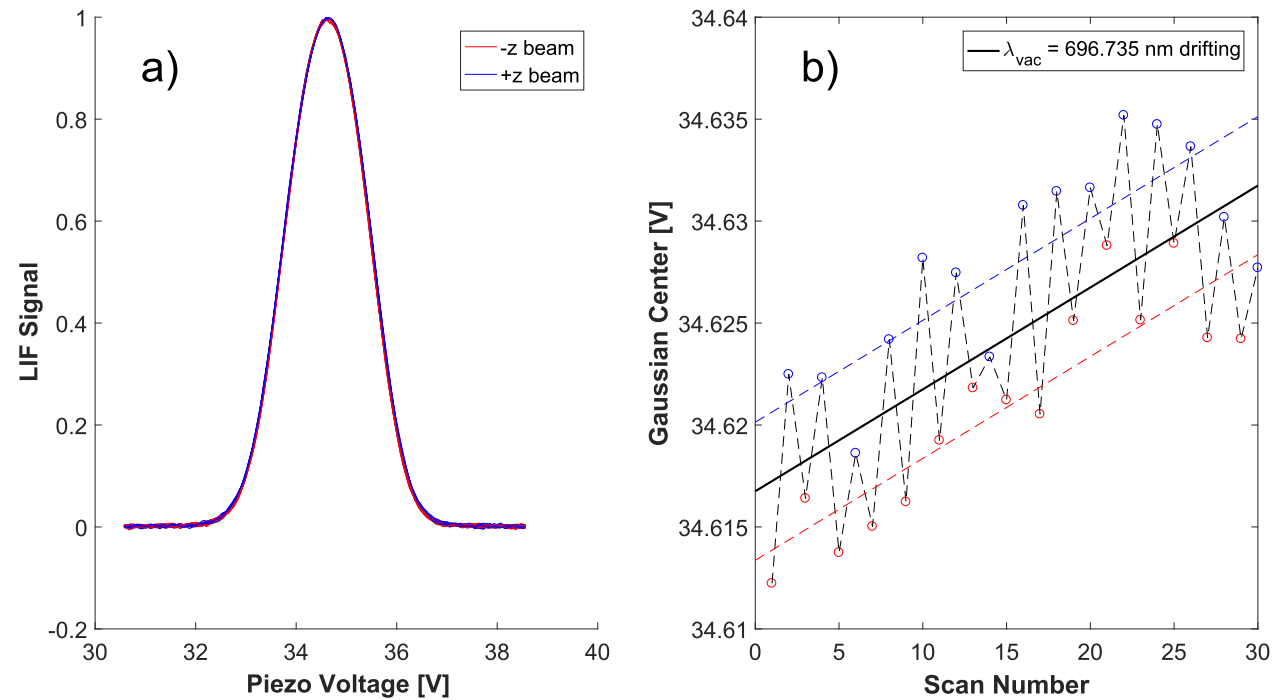

FIG. 6. Illustration of how to calculate the flow speed using the alternating counter-propagating beam approach described in steps $1-5$. (a) shows the 30 measured datasets taken during the $T \approx 450 \mathrm{~s}$ procedure. The data are normalized and plotted on the same axes, so it can be compared. There are 15 red and 15 blue traces representing data from when the $-z$ and $+z$ beams are shining, respectively. (b) shows the center of the Gaussian fit from Eq. (4) for each of the datasets. The black dashed line represents the order the data were collected starting with the red dot at scan number 1 and alternating red and blue subsequently. The slope of the best fit lines represents the drift of the laser itself and the separation of the lines represents the flow velocity. The solid black line between the dashed lines shows how the piezovoltage corresponding to $\lambda_{v a c}$ $=696.735 \mathrm{~nm}$ changes over the 30 measurements due to laser drift.

therefore, piezo voltage is a natural way to look at the data because the diode laser is directly tuned by this parameter. (ii) Plotting the center piezo voltage of each Gaussian fit shows how the diode laser is drifting, whereas plotting the LIF signal vs wavelength would obscure this important complication. (iii) It takes all 30 measurements and their center piezo voltage to separate the drift out of the measurements and obtain an accurate absolute calibration that can be used to calculate the flow speed as will be demonstrated next.

The data in Fig. 6 shows how this repeating, alternating counter-propagating beam approach allows for the flow measurement even when the laser drift is non-negligible. First, the laser drift is determined by taking all 30 data points (red and blue) in Fig. 6(b) and fitting them to a single line $y=m x+b$. The slope of this line $m$ represents the drift of the laser wavelength. Then, the individual beam data are both fit to their own lines, shown by the red and blue dashed lines, where the slope of each best-fit line is forced to be the same slope as the linear best fit of all 30 data points $m$ representing the laser drift. In this prescription, the vertical distance between the two fits, i.e., the y-intercepts, provides the absolute calibration required to calculate the flow speed. Thus, the laser drift (slope of the two lines) and the signed flow velocity (distance between the two lines) can both be determined individually from Fig. 6(b).

The uncertainty associated with the flow measurement is found by examining each pair of consecutive $-z$ and $+z$ beam LIF measurements together. The difference in volts between the center of the first Gaussian for the $-z$ beam and the first Gaussian for the $+z$ beam is found. Then, the difference for each subsequent pair is found. The standard deviation of the 15 differences becomes the error bar on the flow measurement.
The enumerated procedure finds the absolute calibration. The vertical distance between the blue and red dashed lines in Fig. 6(b) is measured to be $\Delta V=0.0068 \pm 0.0030$ piezo volts. The piezo voltage halfway between the shifted peaks, illustrated by the solid black line in Fig. 6(b), corresponds to $\lambda_{v a c}=696.735 \mathrm{~nm}$. Applying the calibration factor from Toptica, the frequency shift between the two peaks is then $\Delta f=\Delta V \times\left(\frac{21 \times 10^{9}}{39.2}\right)=3.63 \mathrm{MHz}$. This gives $v$ $=\Delta f \lambda_{\text {vac }} / 2=1.3 \mathrm{~m} / \mathrm{s} \pm 0.6 \mathrm{~m} / \mathrm{s}$ flow speed. The Doppler shift from this flow changes the wavelength of the center of the peak by $3 \mathrm{fm}$ $=3 \times 10^{-15} \mathrm{~m}$.

\section{2D plane scan}

A two-nozzle setup is used to enable the measurement of the $v_{x}$ and $v_{z}$ flow velocity vector components. Figures $3(\mathrm{a})$ and $3(\mathrm{~b})$ show the coordinate system and the origin of the stepper motor axes for scanning in three dimensions. Nozzle 1 is located at $(-4125,0,0)$ and nozzle 2 is located at $(4125,0,0)$ in stepper motor coordinates where 1600 stepper motor coordinates correspond to $2.5 \mathrm{~mm}$, so one motor step is $1.5625 \mu \mathrm{m}$. The stepper motors are computer controlled, so high spatial precision is obtained.

The PMT and the two fibers with collimators providing the two counter-propagating laser beams were mounted on a single rigid moveable structure. This rigid structure was moved in three dimensions by stepper motors controlled by a Labview program. The LIF diagnostic took the same measurement steps described in Sec. IV B 1 at each of 63 positions on a grid in the $x z$ plane. The data shown in Fig. 6 were taken at $(x, y, z)=(-3000$, $0,3000)$ in stepper motor coordinates, which correspond to $\mathbf{r}$ $=-4.6875 \hat{x}+0 \hat{y}+4.6875 \hat{z}$ in units of $\mathrm{mm}$ from the origin of the 
stepper motor coordinate system shown in Fig. 3. This also corresponds to $1.7587 \mathrm{~mm}$ in the $\hat{x}$ direction and $4.6875 \mathrm{~mm}$ in the $+\hat{z}$ direction from nozzle 1. Figures 7 and 8 show the measured flow speeds as a contour plot for each nozzle. The diagnostic was moved over the $7 \times 9$ grid from $-3000 \leq x \leq 3000$ and $0 \leq z \leq 10000$ or equivalently $-4.6875 \leq x \leq 4.6875$ and $0 \leq z \leq 15.625 \mathrm{~mm}$ at $y=0$. It took approximately $9 \mathrm{~h}$ each to repeat the procedure to deduce flow speed at each of the 63 positions in the grid and create the contours in Figs. 7 and 8.

We now describe how an imposed symmetry created by a prescribed experimental setup enables determination of both in-plane components of the flow velocity.

Consider the $1.3 \mathrm{~m} / \mathrm{s}$ flow shown in Fig. 6 and the contour plots in Figs. 7 and 8. The flow speeds in these figures are flow speeds projected in the direction of the laser beam because LIF can only measure flow parallel to the laser beam. The data in Fig. 6 thus indicate $1.3 \mathrm{~m} / \mathrm{s}=\mathbf{v}_{1}(-3000,0,3000) \cdot \hat{z}$ because the laser beam propagates in the $\hat{z}$ direction. Likewise, the data that make up the contours of Figs. 7 and 8 are contours of $\mathbf{v}_{\mathbf{i}}(x, y, z) \cdot \hat{z}$, where $i=1$ or 2 , respectively.
Figure 9 shows the two-nozzle geometry in detail. Consider measuring $1.3 \mathrm{~m} / \mathrm{s}=\mathbf{v}_{\mathbf{1}}(x=-3000, y=0, z=3000) \cdot \hat{z}$ from nozzle 1 . The actual velocity vector of the argon atom is illustrated by the purple arrow in Fig. 9, but the LIF diagnostic only measures the $\hat{z}$ component, which is the green arrow and completely misses the orthogonal red $\hat{x}$ component. The key symmetry in Fig. 9 is that the flows from nozzle 1 and nozzle 2 are simply rotated by $90^{\circ}$ with respect to each other. The two measuring positions denoted by the two black dots in Fig. 9 are displaced from their respective nozzle by the same vector. This means that the purple arrows (flow velocity) have the same magnitude, but their orientation differs by $90^{\circ}$. The red and green velocity components are similarly the same magnitude. By closing nozzle 1 and opening nozzle 2, the red component is measured instead of the green, which is now orthogonal. Thus, with two nozzles $90^{\circ}$ offset and one beam, LIF can measure two components of the flow speed, $v_{x}$ and $v_{z}$, from a single nozzle.

The second component of the flow velocity is obtained by rotating the $\{z, x\}$ coordinates of the nozzle 2 data by $90^{\circ}$ in the counter clockwise direction. Figure 10 shows arrows made from the nozzle 1
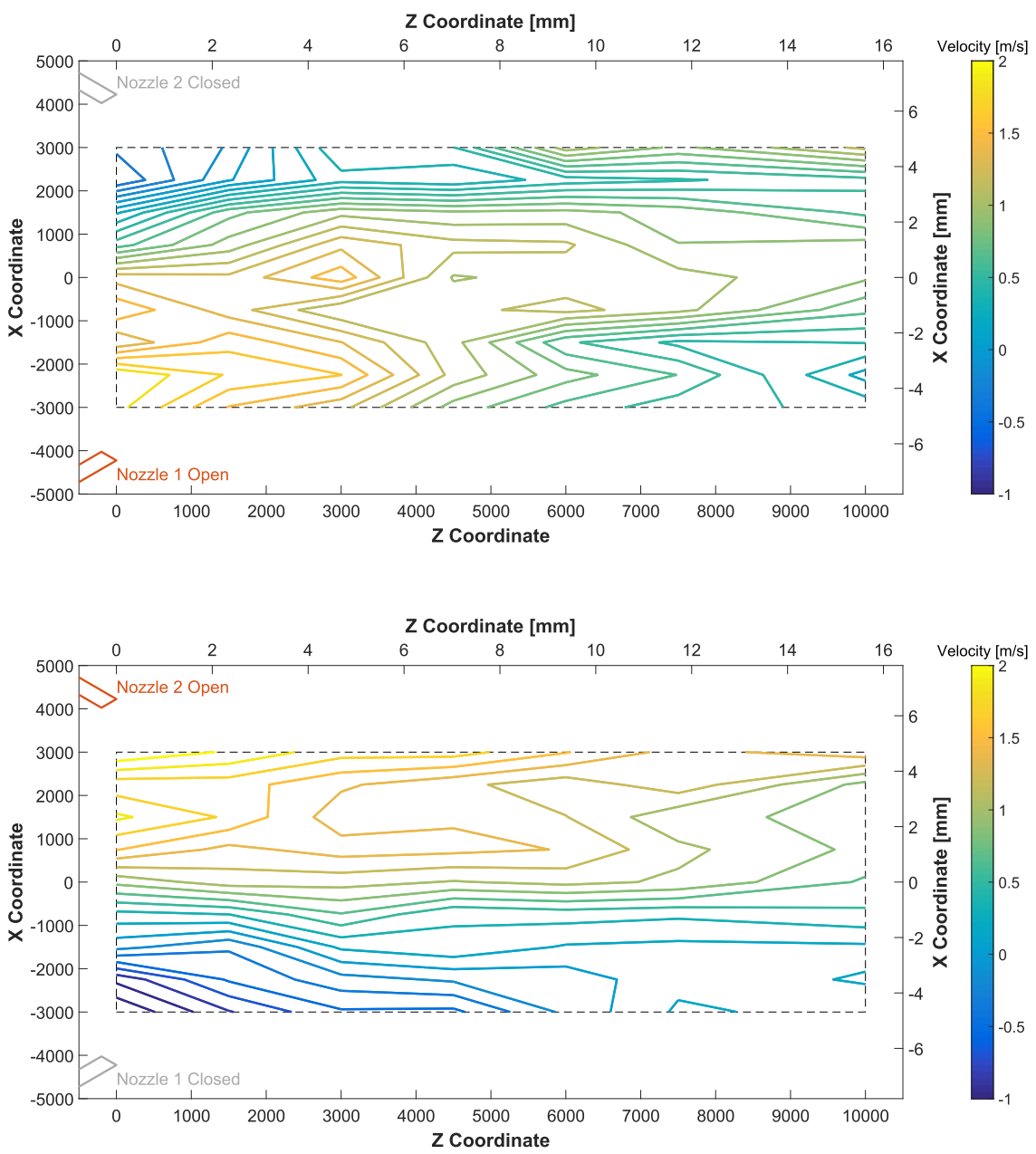

FIG. 7. Measured $\mathbf{v}_{1}(x, y, z) \cdot \hat{z}$ flow contours for nozzle 1 located at $(0,-4125)$ with nozzle 2 closed. It took approximately $9 \mathrm{~h}$ to measure the data needed to create this contour plot.
FIG. 8. Measured $\mathbf{v}_{2}(x, y, z) \cdot \hat{z}$ flow contours for nozzle 2 located at $(0,+4125)$ with nozzle 1 closed. It took approximately $9 \mathrm{~h}$ to measure the data needed to create this contour plot. 


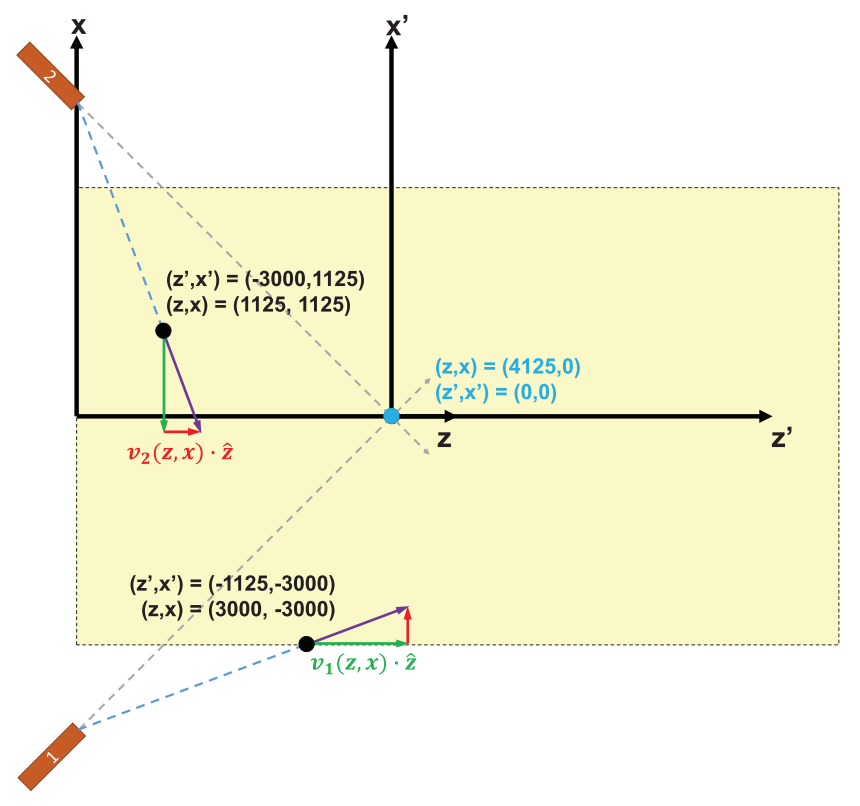

FIG. 9. A to-scale illustration of the rotational symmetry between the nozzles using stepper motor coordinates. Measurements are made in the yellow rectangular region. The origin of the $\left(z^{\prime}, x^{\prime}\right)$ axes sits at $(z, x)=(4125,0)$ and represents the point of rotational symmetry for the nozzles. Each of the measurement positions denoted by black circles is the same vector from its respective nozzle. This symmetry means that the purple flow velocity vector from each position is the same, simply rotated by $90^{\circ}$.

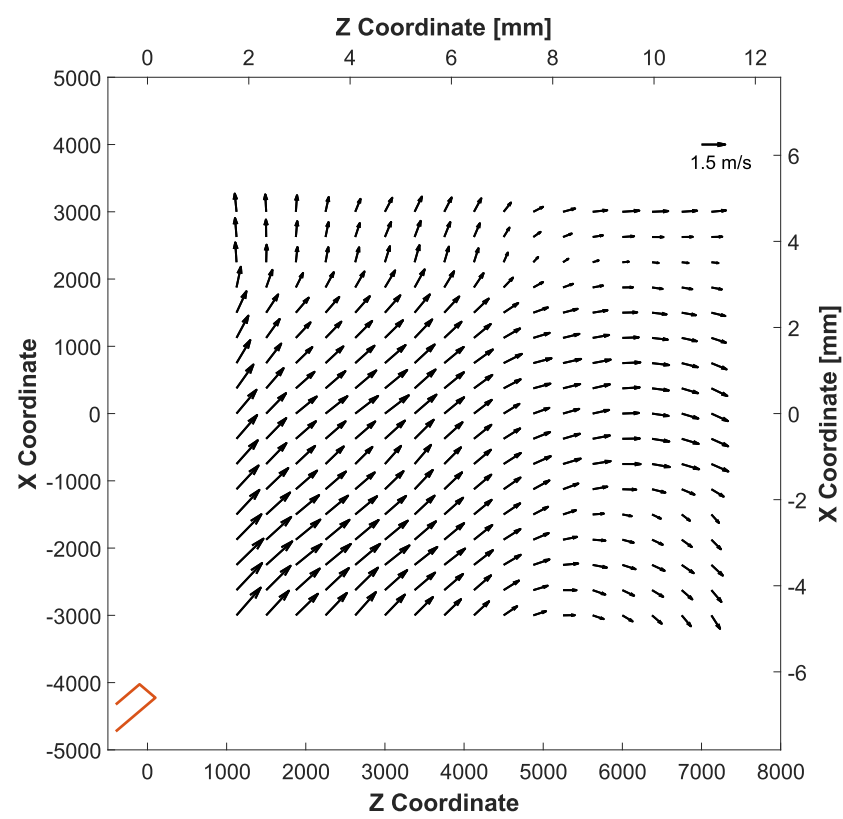

FIG. 10. Two-dimensional in-plane flow velocity measured using the rotational symmetry between the nozzles in the $x z$ plane at $y=0$ represented by arrows with a scale bar in the upper right corner providing a reference. The brown outline near $(0,-4125)$ represents nozzle 1 , the source of this flow. $v_{z}$ data and the nozzle $2 v_{x}$ data where the coordinates from nozzle 2 have been rotated to the nozzle 1 basis. The brown outline represents the location of nozzle 1 . It took $9 \times 2=18 \mathrm{~h}$ to measure the data to create the velocity arrows in Fig. 10 .

The nozzle orientation in the $x z$ plane and the geometry of the electrodes lead us to believe that $v_{y} \ll v_{x}, v_{z}$. The velocity resolution of the LIF diagnostic is exhausted when trying to explore the out-of-plane $y$ component of the flow velocity by assuming an incompressible flow and solving for $\frac{\partial v_{y}}{\partial y}$ in $\nabla \cdot \mathbf{v}=0$. No conclusions are able to be drawn about $v_{y}$.

\section{How flow speed changes with parameters}

The arrows in Fig. 10 clearly show that the flow speed is peaked near the nozzle and decreases as the LIF diagnostic moves away in $x$ and $z$. Changing the rate of inflowing gas by varying the leak valve setting and changing the rate of outflowing gas by partially valving off the turbopump are two ways to vary the neutral argon flow speed in the plasma measured by the LIF diagnostic at a given position. Chamber pressure is determined by an equilibrium between the inflow rate determined by the leak valve and the outflow rate determined by the pumping efficiency of the turbopump. Altering either changes the pressure.

First, one can vary the inflow rate of gas into the chamber by opening or closing the mechanical leak valve. By restricting the flow through the leak valve and holding chamber pressure constant by partially valving off the turbopump, it is seen that the measured flow speed drops by a factor of about three from $4.6 \mathrm{~m} / \mathrm{s}$ to $1.6 \mathrm{~m} / \mathrm{s}$.

Second, one can change the pressure in the chamber while holding the inflow leak valve setting constant and partially opening the valve to the turbopump. As the chamber pressure drops from 330 mTorr to 90 mTorr, it is seen that the flow speed measured by the LIF diagnostic increases from $1.7 \mathrm{~m} / \mathrm{s}$ to $4.6 \mathrm{~m} / \mathrm{s}$.

Third, it is also seen that physically reversing the direction of the flow that is projected into the direction of the laser beam path produces very similar results. The only difference is that the sign of the measured flow velocity has reversed.

Figure 11 shows the second and third trends graphically. In the figure, flow is measured, while the nozzles introduce flow in opposite directions along the axis of the LIF diagnostic separately. Unsurprisingly, the diagnostic measures flow in the opposite direction (sign) depending on which nozzle is used. Varying the pressure in the chamber by partially valving off the turbopump while holding the inflow setting constant produces flow speeds of approximately the same amplitude regardless of which nozzle is used. The only difference is the sign. The negative flow datapoint at 333 mTorr with the particularly large error bar was made from data with considerably more laser drift than the other measurements.

\section{Mean free path for collisions}

Consider the mean free path for collisions of inflowing neutral argon atoms,

$$
\lambda_{m f p}=\frac{\kappa T}{\sqrt{2} \pi d^{2} p} .
$$

Doubling the van der Waal's radius gives the effective diameter $d$ of an argon atom; $d=376 \mathrm{pm} .{ }^{21,22}$ For a room temperature plasma at $T=300 \mathrm{~K}, \lambda_{m f p}$ in meters is inversely related to pressure 


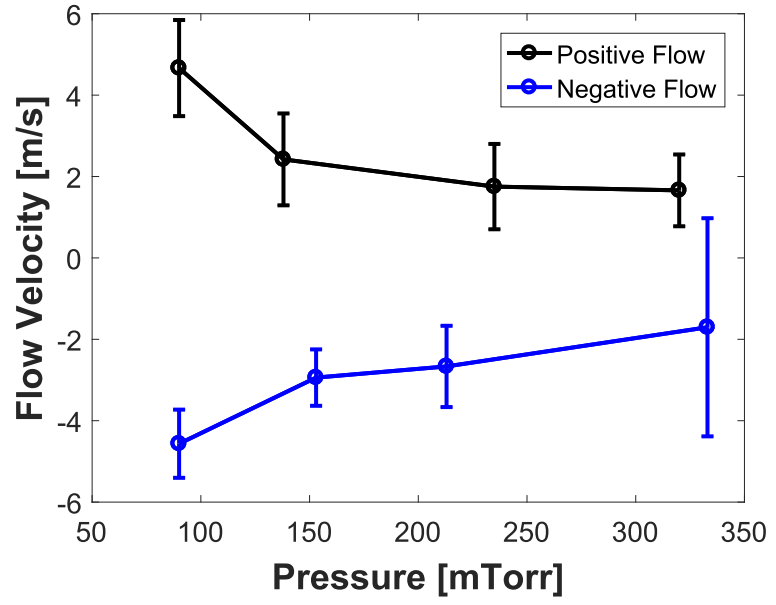

FIG. 11. Measured flow speed with error bars as a function of chamber pressure. Inlet flow is held constant and pressure is varied by partially valving off the turbopump. The positive and negative flows are made from nozzles producing flow in opposite directions projected on the LIF beams.

by $\lambda_{m f p}=0.049 / p$, where $p$ is in mTorr. For $p=100$ mTorr, $\lambda_{m f p}$ $=0.5 \mathrm{~mm}$.

The flow speed decreases as the LIF diagnostic is moved away from the nozzle because the inflowing argon atoms have to travel through more mean free paths before they reach the beam. This means that they have thermalized more and have less of a bulk flow. By holding the nozzle and LIF diagnostic in the same position and increasing the background pressure, the mean free path for collisions is reduced and the inflowing argon atoms have to travel more mean free paths to get to the same position. This means that they are more thermalized, so the measured flow speed is less. When the nozzles were outside the plasma, the argon flow had to travel through $\sim 3 \mathrm{~cm}$ of the plasma to reach the laser beams, which is more than 30 mean free paths, so the flow was likely fully thermalized with no bulk flow left. This is presumed why the initial effort to measure flow with the nozzles in Fig. 2(a) configuration failed.

\section{Resolving details smaller than the natural linewidth}

One subtle issue is that the linewidth of the $696.735 \mathrm{~nm}$ transition in neutral argon is $\Delta f=5.6 \mathrm{MHz}$. ${ }^{10}$ One might therefore presume as in Ref. 3 that the minimum resolvable flow speed is then $v_{\text {min }}=\Delta f \lambda_{v a c}=3.9 \mathrm{~m} / \mathrm{s}$. One would be troubled that all the flow speeds presented in this paper have $v<v_{\min }$ and that we are resolving speeds of a fraction of a meter per second. However, this would only be an issue if our conclusions were based on single point measurements where one would experience the full linewidth as uncertainty. This is not the case for the methods described here because data are taken at many different wavelengths and are fitted to a Gaussian shape. The peak of the Gaussian can be resolved to much less than the transition linewidth because determining the peak involves averaging over a large number of data points.

\section{Relative density and attenuation coefficient measurements}

Figures 12(a)-12(d) show that the measured LIF signal changes when translating the LIF diagnostic along the beam axis $\pm \hat{z}$. Figure 12(a) shows LIF data measured when the $-z$ beam traverses the plasma. Each color trace superimposes 15 measurements made at each of the seven $z$ positions shown in the color bar on the right. Likewise, Fig. 12(c) shows the same 15 measurements for each color when the $+z$ beam traverses the plasma. Figures 12(b) and 12(d) plot the average temperature deduced from the 15 LIF measurements at each position in Figs. 12(a) and 12(c), respectively. Statistical uncertainty error bars were calculated from the standard deviation of the temperature deduced from the 15 LIF measurements at each position. These bars are not shown in Figs. 12(b) and 12(d) because they are smaller than the colored circle data point markers.

These measurements from the $+z$ and $-z$ beams show a consistent approximately factor of two difference in the LIF signal amplitude between $z=0$ and $z=15.625 \mathrm{~mm}$, independent of which beam is used. This indicates that there is a neutral argon density gradient along the measurement axis such that the density increases toward large $z$ where the LIF signal is strongest.

The Doppler widths of the detected LIF signals also change with $z$. Both Figs. 12(b) and 12(d) show that the deduced temperature of the neutral argon atoms in the plasma increases as the pump beam is increasingly attenuated. Figure 12(b) shows that the deduced temperature of the neutral argon atoms measured by the $-z$ beam increases toward small $z$. Similarly, Fig. 12(d) shows that the deduced temperature of the neutral argon atoms measured by the $+z$ beam increases toward large $z$. In both cases, the deduced temperature increases with the increase in beam travel through the plasma and therefore increasing attenuation.

The opposite temperature gradients deduced in Figs. 12(b) and 12 (d) suggest that not all wavelengths of light are absorbed equally by the plasma. Moreover, it suggests a broadening mechanism whereby the deduced temperature is increased as the beam is differentially absorbed by the plasma. This mechanism referred to as "absorption broadening" is a result of the attenuation coefficient $\kappa$ at the center of the peak being greater than at the wings. Mathematically, this means that the attenuation coefficient $\kappa=\kappa(\lambda)$ and $\kappa\left(\lambda_{0}\right)>\kappa(\lambda)$ for $\lambda \neq \lambda_{0}$.

We now present an analysis of what is happening in Fig. 12. This analysis will not explain everything but provides a strong foundation. $A_{ \pm}(z)$, the amplitude of the LIF signal at position $z$ from the \pm beam, is influenced proportionally (up to saturation) by (i) $n(z)$, the density of the target species at position $z$, and (ii) $P_{ \pm}(z)$, the intensity of the \pm beam at position $z$. The relationship between pump beam intensity and distance $z$ traveled through a plasma of uniform density is $P_{ \pm}(z)=P_{ \pm}(0) e^{-\kappa z}$. More specifically, the attenuation coefficient $\kappa=\kappa(z, \lambda)=\alpha(\lambda) n(z)$ is proportional to neutral argon density and is also a function of wavelength via the $\alpha(\lambda)$ term. Therefore, the pump beam amplitude at a given wavelength traveling through a plasma with non-uniform density is $P_{ \pm}(z, \lambda)=P_{ \pm}(0, \lambda) e^{-\int_{0}^{z} \alpha(\lambda) n(z) d z}$. Considering the direction each beam is traveling, it is proposed that for a given wavelength, the two functions

$$
A_{+}(z)=A_{+}(0) n(z) e^{-\alpha \int_{0}^{z} n\left(z^{\prime}\right) d z^{\prime}}
$$



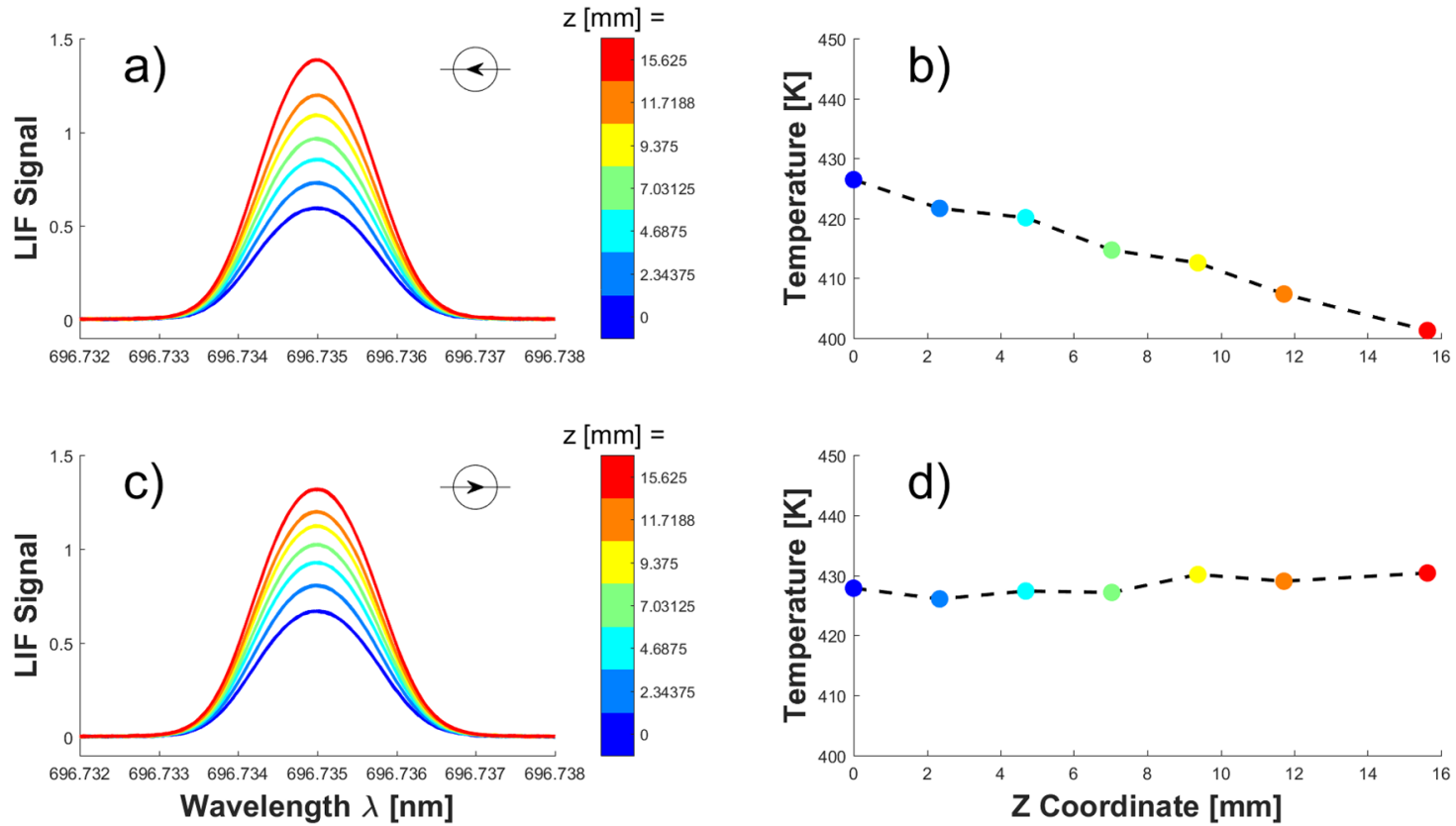

FIG. 12. (a) and (c) show the LIF signal measured by the PMT as the LIF diagnostic is moved along the beam axis from $z=0$ to $z=15.625 \mathrm{~mm}$ for the $-z$ beam and the $+z$ beam, respectively. 15 individual datasets are superimposed in each color on both plots, showing the great reproducibility of the diagnostic. (b) and (d) plot the temperature deduced from the data in (a) and (c), respectively, with matching color. Each data point in (b) and (d) is found by taking the average of the 15 deduced temperatures from the 15 datasets plots on (a) or (c) in the same color. Error bars are found by taking the standard deviation of the 15 deduced temperatures. These statistical uncertainty error bars are not shown for the deduced temperatures plotted in (b) and (d) because the data point markers (colored circles) themselves are larger than the error bars. For example, the blue point at $z=0$ in (d) is $T=427.8 \mathrm{~K} \pm 1.1 \mathrm{~K}$.

$$
A_{-}(z)=A_{-}(0) n(z) e^{-\alpha \int_{z}^{0} n\left(z^{\prime}\right) d z^{\prime}}
$$

quantify how the LIF signal varies along the beam axis for each beam. All multiplicative constants are included in the $A_{ \pm}(0)$ terms. A consequence of this is that $n(z)$ is effectively a relative density at each position and not an absolute density.

It is important to explain why the limits of integration are switched between Eqs. (7) and (8). Equation (7) is integrated from $z^{\prime}=0$ to $z^{\prime}=z$ because the $+z$ beam is exponentially attenuated as it travels in the $+z$ direction from 0 to $z$. The $-z$ beam travels in the opposite direction, so it is exponentially attenuated as it travels in the $-z$ direction from $z^{\prime}=z$ to $z^{\prime}=0$, which is why Eq. (8) has its limits of integration switched. Equations (7) and (8) can be written more compactly as

$$
A_{ \pm}(z)=A_{ \pm}(0) n(z) e^{\mp \alpha \int_{0}^{z} n\left(z^{\prime}\right) d z^{\prime}} .
$$

Figure 13(a) plots the seven signal amplitudes measured at the center wavelength at each position $z$ in Fig. 12(a), i.e., $A_{-}(z)$, and Fig. $12(\mathrm{c})$, i.e., $A_{+}(z)$, on the same set of axes. The amplitude plotted at each point is the average amplitude of the 15 LIF measurements at each position in Figs. 12(a) and 12(c). Error bars represent the standard deviation of each set of 15 amplitude measurements. $z=0$ is the reference position for each beam, so $n(z=0)=1$ by definition.

For the $+z$ beam, the signal amplitude $A_{+}(z=0)=A_{+}(0) n(0) e^{0}$ $=A_{+}(0)=0.6711$. The $A_{+}(z)$ selection in Eq. (9) provides a relationship between each of the seven $\left[z, A_{+}(z)\right]$ pairs plotted on Fig. 13(a) in blue, where $n(z)$ and $\alpha$ remain unknown. Similarly, for the $-z$ beam, the signal amplitude $A_{-}(z=0)=A_{-}(0) n(0) e^{0}$ $=A_{-}(0)=0.5969$. The $A_{-}(z)$ selection in Eq. (9) provides a relationship between each of the seven $\left[z, A_{-}(z)\right]$ pairs plotted on Fig. 13(a) in red where $n(z)$ and $\alpha$ again remain unknown.

These relationships found using Eq. (9) provide 14 equations for 8 unknowns. $n(0)=1$ is trivial by definition, so there are effectively 12 equations and 7 unknowns: $n(z)$ for each non-zero $z$ position and $\alpha$. The relative density $n(z)$ is obtained by multiplying the obtained $A_{+}(z)$ and $A_{-}(z)$ relationships for each $z$ together. The exponential integrated attenuation terms cancel exactly, so the relative density is $n(z)=\sqrt{\frac{A_{+}(z) A_{-}(z)}{A_{+}(0) A_{-}(0)}}$, where all four numbers on the right side are known. Relative density $n(z)$ is plotted in Fig. 14. Statistical error from the signal amplitude measurements is propagated through the relative density calculation and found to be negligibly small compared to the size of the data point markers and thus is left off the plot. In Fig. 14, $n(15.625)=2.1425$ means that the density at $z=15.625 \mathrm{~mm}$ is 2.1425 times the density at $z=0$.

With $A_{ \pm}(z), A_{ \pm}(0)$, and $n(z)$ known for each $z, \alpha$ up to each of the six non-zero $z$ positions can be obtained using either the + or selection in Eq. (9). Calculating the average and standard deviation of the six obtained $\alpha$ values gives $\bar{\alpha}=5.2 \times 10^{-6} \pm 2.3 \times 10^{-7}$.

Figure 13(b) shows the LIF signal amplitudes after they have been normalized by $n(z)$ (the relative density factors plotted on 


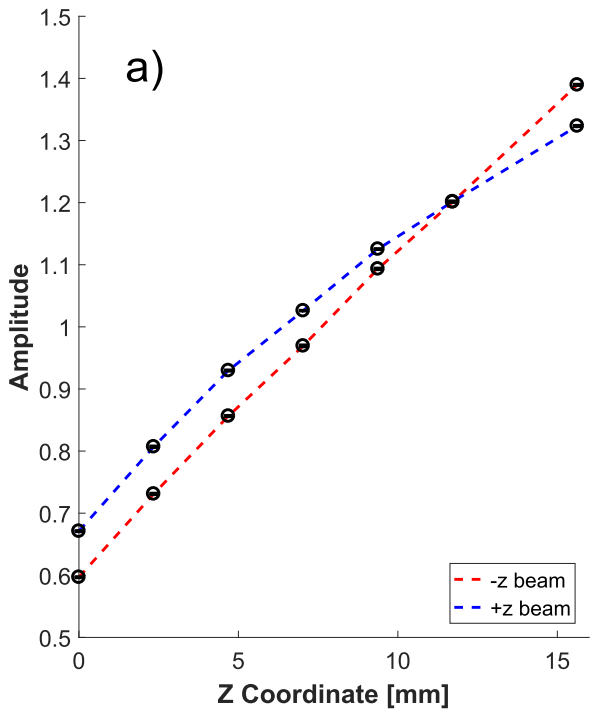

Fig. 14) and normalized again such that the largest signal measured for each beam is one. The error bars represent the standard deviation error propagated through the calculation to this point.

Instead of normalizing only the peak amplitude of the LIF signal, we now normalize each entire LIF measurement from Figs. 12(a) and 12 (c) by $n(z)$ plotted in Fig. 14 . This process does not affect the deduced temperature because the temperature does not scale with the signal amplitude.

The 15 resulting normalized datasets for the $+z$ beam and the $-z$ beam are each averaged into a separate dataset and fit to a Gaussian. Because the beams are counter-propagating and there is a slow flow, the Gaussian fits all have slightly different centers. This is

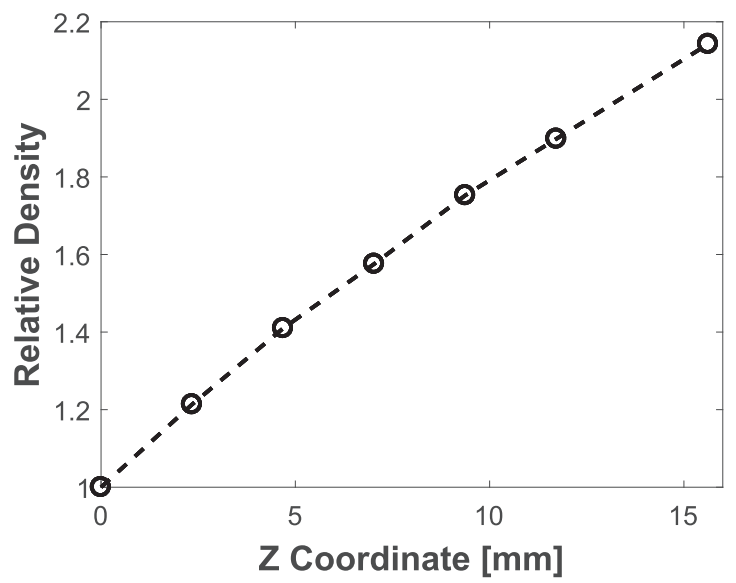

FIG. 14. Deduced argon neutral relative density $n(z) . n(15.625)=2.1425$ means that the density at $z=15.625 \mathrm{~mm}$ is 2.1425 times the density at $z=0$. Error bars are not shown in the figure because the error bars associated with the statistical uncertainty in the density calculation are smaller than the data point markers (circles). manually corrected by shifting all the Gaussian fits to have their center at $\lambda_{v a c}$. Shifting the Gaussian does not affect the deduced temperature because the temperature does not depend on the center location. Figure 15 shows normalized data on the same axes after averaging and correcting for slow flow. The solid lines are from the $-z$ beam and the dashed lines are from the $+z$ beam.

The density gradient is not a factor affecting the data in Fig. 15, in contrast to the raw data in Figs. 12(a) and 12(c). In Fig. 15 for the $-z$ beam, the largest amplitude is at $z=15.625 \mathrm{~mm}$, whereas for the $+z$ beam, the largest is at $z=0$. The largest amplitude for each beam is thus seen where the beam is incident on the plasma and the LIF signal amplitude decreases as each beam is attenuated by traveling through plasma.

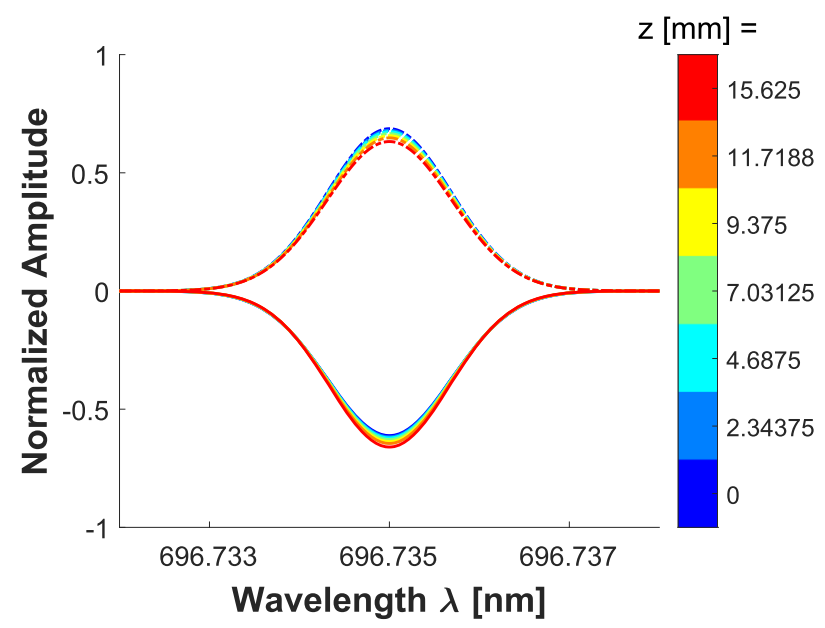

FIG. 15. LIF signals from Figs. 12(a) and 12(c) normalized by relative density $n(z)$ The solid lines are from $-z$ beam (inverted) and the dashed lines from the $+z$ beam. 
The last step now is to solve for the wavelength dependent part of the beam attenuation $\alpha(\lambda)$. This is done by dividing the wavelength dependent LIF signal measurements at two different positions in Eq. (9) to obtain

$$
\alpha_{ \pm}=\frac{\mp \log \left(\frac{A_{ \pm}\left(z_{2}\right) n\left(z_{1}\right)}{A_{ \pm}\left(z_{1}\right) n\left(z_{2}\right)}\right)}{\int_{0}^{z_{2}} n(z) d z-\int_{0}^{z_{1}} n(z) d z} .
$$

Figure 16 shows $\alpha(\lambda)$ for the $-z$ beam and the $+z$ beam data calculated using Eq. (10) at each wavelength $\lambda$. There are now six rather than seven $\alpha_{ \pm}$curves because one of the seven positions is used as the reference and the other six are used to calculate $\alpha$ using the +or - selection in Eq. (10). The plotting domain is reduced because the LIF signal peak is largely contained in this domain, and outside this domain away from the peak, $\alpha$ does not have a physical meaning. The six curves for $\alpha$ from the $-z$ beam and $+z$ beam agree within the measurements of each beam and the peak magnitude of $\alpha$ shows excellent agreement across beam measurements.

The $\alpha_{ \pm}$curves in Fig. 16 quantify absorption broadening. They show that the attenuation coefficient of the pump beam $\alpha$ is a function of wavelength around the peak $\lambda_{v a c}$ absorption. Both plots show that the plasma is the most opaque absorber at $\lambda_{v a c}$ and becomes marginally less opaque away from $\lambda_{v a c}$ on the peak. This means that the detected LIF signals will get distorted as more pump beams are attenuated at $\lambda_{v a c}$ such that the wings of the spectra see increased signal amplitude relative to the center resulting in artificially increasing deduced temperatures.

It is not clear why $\alpha_{-}(\lambda)$ is more sharply peaked than $\alpha_{+}(\lambda)$, or equivalently, why absorption broadening is stronger on the $-z$ beam, and as a result, the temperature gradient for the data measured with the $-z$ beam is larger than the temperature gradient for the $+z$ beam. Figures 12(b) and 12(d) picture this as Fig. 12(b) shows a larger temperature gradient than that shown in Fig. 12(d). It was postulated that this effect was due to the optical fibers having a finite opening angle $\left(\sim 0.3^{\circ}\right)$ as opposed to the light being collimated to infinity, but attempts to correct for this did not make the $\alpha_{ \pm}$peaks match.

While the model developed has improved the understanding of how the laser beam is attenuated by the plasma, the fundamental reason for this difference in the strength of the absorption broadening mechanism for the $-z$ and $+z$ beams remains unknown. Possible explanations for the differing gradients are that there is still some power broadening or there is a hysteresis-like effect from the differential damping of the laser beam - the $-z$ beam first travels through dense plasma and then through less dense plasma, whereas the $+z$ beam first travels through less dense plasma and then through higher density plasma.

With an absorption spectroscopy diagnostic, the only measurement obtained would be a line-averaged measurement of this whole effect where density gradient and absorption broadening the temperature would remain unknown.

\section{Flow impact on ice grains}

To investigate how the inflowing argon atoms affect the waterice grains, the nozzles shown in Fig. 2 are set into an intermediate configuration where nozzle 2 is inside the plasma as shown in Fig. 2(b) and nozzle 1 is outside the plasma as shown in Fig. 2(a). Argon gas flows continuously into the chamber through nozzle 1 throughout the experiment to sustain the plasma, and water vapor flows in when allowed to create the ice grains. The goal is to see how toggling nozzle 2, thus creating a new flow in a different direction, affects the ice grains. After the electrodes have cooled down, water vapor is allowed to flow into the chamber for $20 \mathrm{~s}$ through nozzle 1 . The water vapor inlet is then closed and a cloud of ice grains forms and grows in the plasma. A horizontal sheet of HeNe laser illuminates a plane of ice grains. The effect of a gas flow on the ice grains can be seen by toggling nozzle 2 on and off.

Ice grain motion was recorded using a Dalsa Falcon VGA300 fast movie camera outfitted with a Nikon $24 \mathrm{~mm}$ lens and a $632 \mathrm{~nm}$
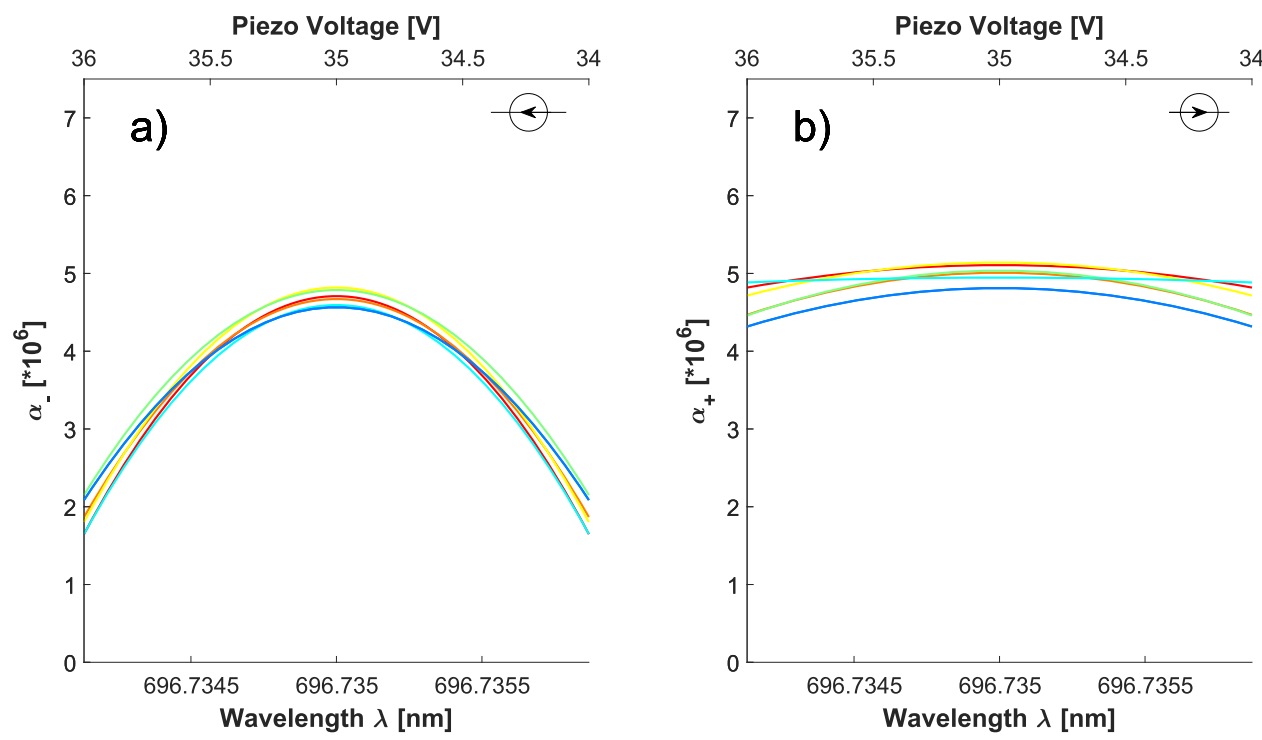

FIG. 16. $\alpha$ solved using Eq. (9) for the $-z$ beam selection on (a) and the $+z$ beam selection on (b). 
HeNe line filter. The lens attaches to the camera via a Nikon to Cmount adapter. The line filter only allows HeNe laser light scattered off the ice grains to be imaged by the camera. The camera is located approximately $30 \mathrm{~cm}$ from the center of the plasma at a position slightly below the plane of the plasma. Figure 17 shows the view from this position by superimposing two images. The cyan-scaled visible-light image shows the physical setup with nozzle 2 on the left of the frame. The side of the bottom electrode is visible as is the flat disk of the top electrode. The ice grain cloud is photographed and superimposed in red. The near edge of the grain cloud is the edge closer to nozzle 2. The far edge is on the far side away from nozzle 2. The high frame rate of this camera (250 FPS used) allows for multiple frames to be taken when the flow from toggling nozzle 2 interacts with the ice grains. The Dalsa camera takes 8-bit grayscale images.

Figure 18 (multimedia view) (the movie can be found in the online version) shows the motion of the grain cloud as it is exposed to a flow through nozzle 2. The movie was recorded at 250 FPS and played back in this video at 10 FPS, a factor of 25 slow-down. The video shows the dramatic effect on the ice grain cloud from toggling on the neutral gas flow injected through nozzle 2 .

Figure 19 shows a set of three artificially colored images from the movie in Fig. 18 (multimedia view) in the top row with key experimental features outlined including the nozzle connected to the valve that is toggled in white on the left-hand side of the each image and the electrodes in yellow dashed lines. The camera is below the midplane of the plasma, as shown in the bottom row of Fig. 19, which is why it sees the side of the bottom electrode and the full flat disk of the top electrode. The red-scale images were taken with a $\mathrm{HeNe}$ line filter to see only the ice grains. Underneath each ice grain image is a top view sketch of the estimated boundary of the ice grain cloud.

The left image in Fig. 19 is the frame taken at time $t$ when the valve is opened. The middle image was taken at $t+0.076 \mathrm{~s}$ or 19

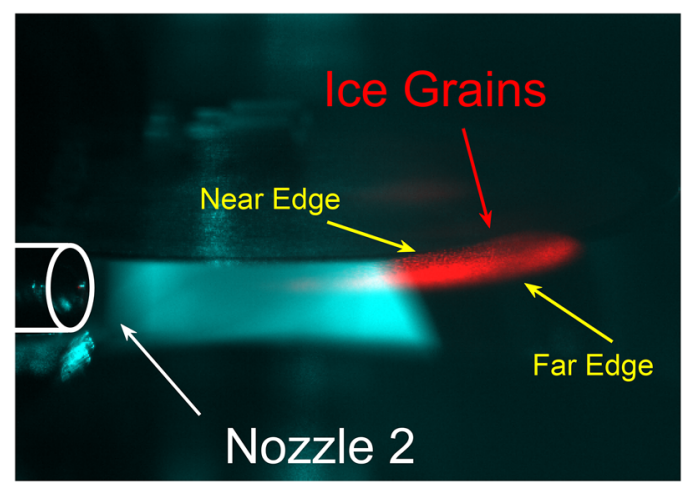

FIG. 17. Superimposed cyan-scale and red-scale images. The cyan-scaled visiblelight image shows the physical setup of the flow experiment with nozzle 2 on the left of the frame. Because the camera is slightly below the plane of the plasma, the side of the bottom electrode is visible as is the flat disk of the top electrode. Plasma exists between the electrodes but no plasma is seen in these images. The ice grain cloud is photographed in a separate red-scale image and superimposed. The near edge of the grain cloud means closer to nozzle 2. The far edge is on the far side.

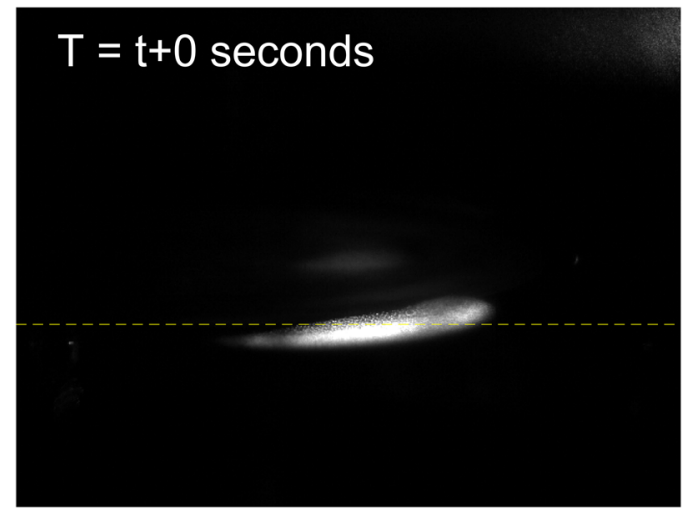

FIG. 18. Ice grain cloud after nozzle 2 is toggled on to allow an injected flow to move the cloud. Image in the text shows the frame at time $t$ when nozzle 2 is toggled on. The movie online shows the effect of the flow on the grain cloud. The movie was originally recorded at $250 \mathrm{FPS}$ and is played back at $10 \mathrm{FPS}$, a factor of 25 slow-down. Multimedia view: https://doi.org/10.1063/5.0006684.1

frames later and the right-hand image was taken at $t+0.3 \mathrm{~s}$ or 75 frames later. The radial $\hat{r}$ motion of the ice grains away from nozzle 2 when it is opened takes place in the first $0.076 \mathrm{~s}$ of motion, and the final $0.224 \mathrm{~s}$ of motion is primarily in the tangential $\hat{\theta}$ direction as illustrated by the unit vectors in the middle sketch on Fig. 19. Nozzle 2 is located on the left-hand side of the image and has diameter $d$ $=6.27 \mathrm{~mm}$. The radial motion appears to cease as the ice grains near the edge of the electrodes and the plasma.

Figure 20 quantifies the motion in the radial direction. The positions of the near and far edges of the ice grain cloud and their speeds are obtained from the video and plotted in Fig. 20(a). They were obtained for each frame by plotting pixel intensity as a function of position along the horizontal yellow dashed line in Fig. 18 and finding the pixel of the intersection between the pixel intensity trace and a selection criterion value.

Nozzle 2 is close to $z=0$ and the flow coming out of it has inplane flow velocity components $v_{x}$ and $v_{z}$ shown in Fig. 10 near the midplane $y=0$. Figure 20(b) shows that when the valve is opened, the near edge of the ice grain cloud at the approximate center $(x$ $=0$ ) moves with speed $v_{z} \approx 0.25 \mathrm{~m} / \mathrm{s}$ away from nozzle 2 and the far edge of the cloud at the approximate center $(x=0)$ moves at $v_{z} \approx$ $0.05 \mathrm{~m} / \mathrm{s}$. The flow speed error bar on Fig. 20(b) is shown vertically on the right side of the plot. It was calculated by finding the distance between the pixel associated with the selection criterion and a $10 \%$ increase in pixel intensity. There is potentially some error from the fact that the camera is not looking at the grain motion from the perpendicular direction. This likely results in measuring a mix of $x$ and $z$ direction motion of the cloud. Nevertheless, this uncertainty is sufficiently small to conclude that there is a dust velocity gradient and that this gradient indicates that the more distant the grains are from the nozzle, the slower they move, which is the same trend seen by neutral LIF in Figs. 7, 8, and 10.

It was challenging to find a global two-variable fit that accurately fits the measured $v_{x}, v_{z}$ flow data in Fig. 10, so instead, Fig. 21 shows an exponential fit to the relevant $v_{z}(x=0, y=0,0 \leq z$ $\leq 15.625 \mathrm{~mm})$ data. The fit is made from the green data points and 

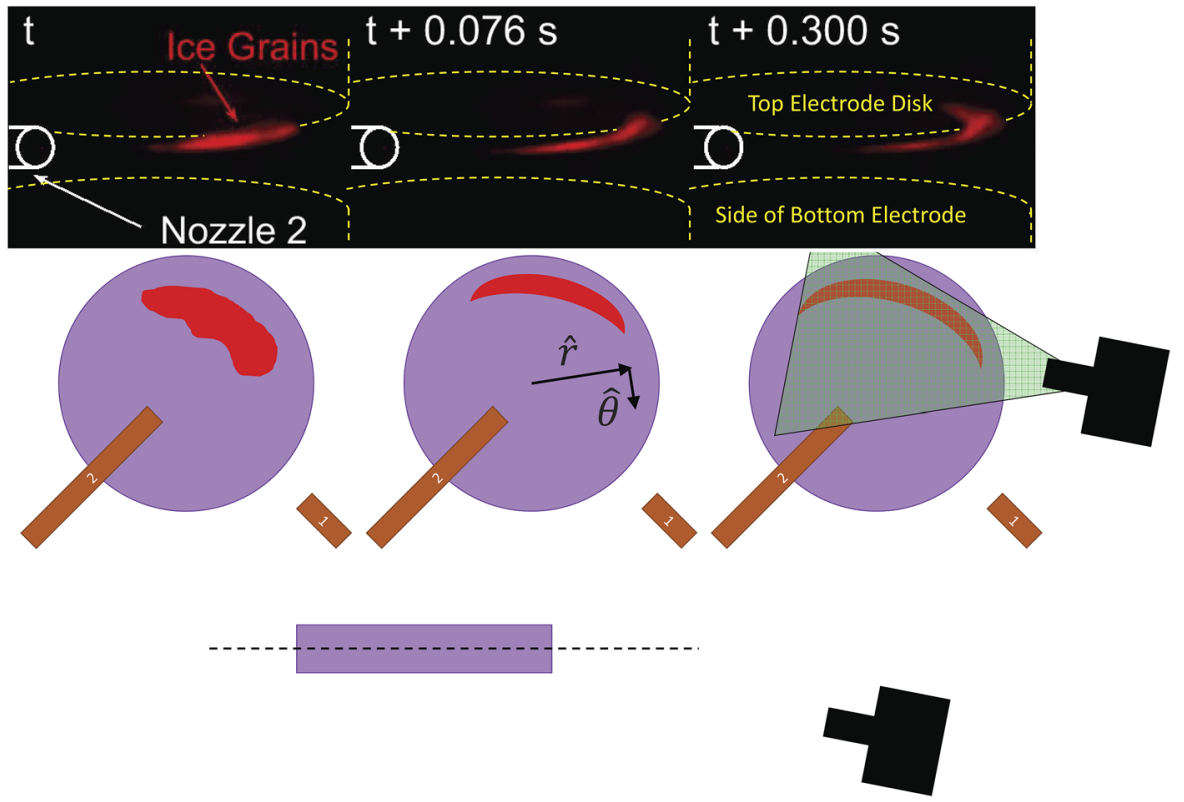

FIG. 19. Top row: artificially colored redscale images of the ice grains inside the Caltech water-ice dusty plasma experiment. Red HeNe laser light scatters off the ice grains and passes into the camera through a line filter, so the ice grains are the only thing visible. The electrodes are outlined in yellow dashed lines and nozzle 2 is outlined in white for perspective. The sequence of images shows how the ice grains move after nozzle 2 is toggled open. Middle row: top view sketches showing the estimated boundaries of the ice grains from each image in the top row. On the right is a sketch of the Dalsa camera showing the approximate field of view. Bottom row: sketch showing that the Dalsa Camera is below the midplane of the plasma. the red $\mathrm{x}$ is excluded as an outlier. Error bars calculated as in Sec. IV B 1 are shown as well. The fit $v_{z}(0,0, z)=1.28 e^{-0.0213 z}$ is found with $R^{2}=0.86$, where $z$ is in $\mathrm{mm}$. Extrapolating this fit to the region where ice grains are present, $23.4375 \leq z \leq 31.25 \mathrm{~mm}(15000 \leq z \leq 20000$ stepper motor coordinates), gives $v_{z}(0,0,23.4375 \mathrm{~mm}) \approx 0.78 \mathrm{~m} / \mathrm{s}$, which is approximately triple the speed of the near edge of the ice grains. Furthermore, $v_{z}(0,0,31.25 \mathrm{~mm}) \approx 0.66 \mathrm{~m} / \mathrm{s}$, which is more than 10x the measured speed of the far edge. The three images in the top row and sketches in the middle row of Fig. 19 show how the grain motion gradient when nozzle 2 is opened causes the ice grain cloud to compress in the $\hat{r}$ direction as the grains move toward the edge and expand in the tangential $\hat{\theta}$ direction. This bulk grain motion suggests that the grains are being held inside the plasma by a sheath force that must oppose the force of the argon streaming toward them and that this opposing force quickly increases toward the edge of the plasma.

An interesting observation is made when nozzle 2 is closed after the grains are in the crescent shape at the edge of the electrodes (the right-hand side images of Fig. 19). When nozzle 2 is closed, the ice grain cloud reverts to its original shape (the left-hand images of Fig. 19). The speed with which this reversion happens is slower. In fact, nozzle 2 can be opened and closed repeatedly with
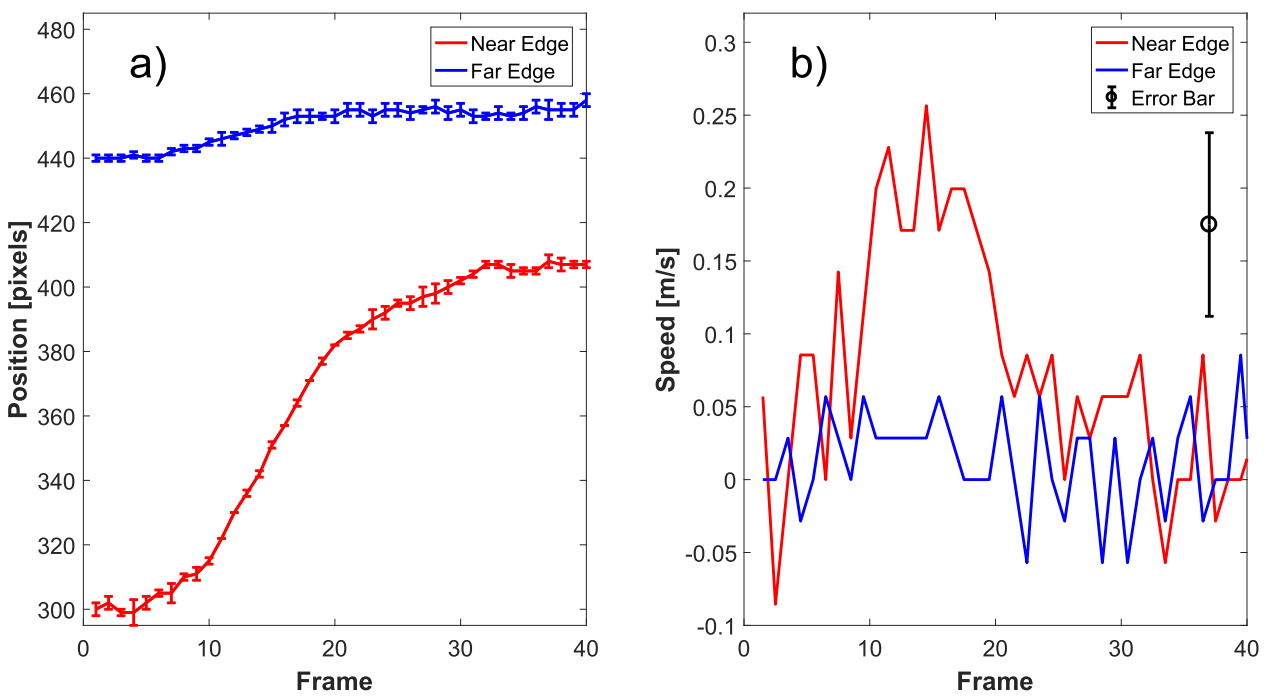

FIG. 20. (a) Plots the position of the near and far edges (to nozzle 2) of the ice grain cloud in each movie frame, while the grains are exposed to the flow from nozzle 2. (b) Plots the speed with which each edge moves. The velocity error bar $( \pm 0.075 \mathrm{~m} / \mathrm{s})$ is shown as the black vertical line on the upper right-hand side of the plot. 


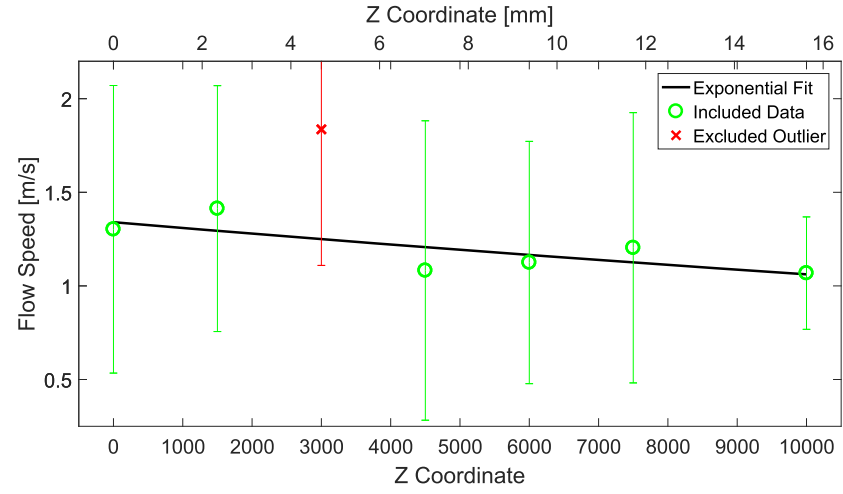

FIG. 21. Measured $v_{z}(0,0, z)$ data denoted by the green o's and the red $x$. These data are also included in Figs. 7 and 10. The green 0 data are fit to an exponential curve. The single red $\mathrm{x}$ is excluded from the fit because it appears to be an outlier.

minimal ice grain losses and the same motion cycle repeats each time.

\section{CONCLUSION}

A specialized new LIF diagnostic has been constructed and operated on the Caltech water-ice dusty plasma experiment in both room temperature dust-free and cold astrophysically relevant dusty conditions. Four motorized degrees of freedom controlled via a Labview computer program allow for automated measurement of temperature and flow speed of the neutral argon species in the three-dimensional plasma volume. The collected data have high signal to noise ratio, in excess of 100 , and are very reproducible. By exploiting the symmetry of the laser beam and nozzle configuration, a single laser beam is able to deduce the two-dimensional in-plane flow velocity vector of the neutral argon species with sublinewidth resolution. Utilizing measurements from both counterpropagating beams, the competing influences of density gradient and absorption broadening were separated and quantified. Neutral flow LIF data compared with the high-speed video of the ice grains exposed to flow reveal how ice grains respond to an imposed flow.

\section{ACKNOWLEDGMENTS}

The authors thank Professor Truell W. Hyde of Baylor University in Waco, TX, for graciously inviting us to use an early version of the LIF diagnostic described here on the PK4 replica experimental setup at CASPER to measure temperature and to attempt (unsuccessfully) to measure flow speeds. We also thank Jorge Carmona Reyes and Kenneth Ulibarri for assistance over 10 days in operating the PK4 replica experimental setup.

We would also like to thank Armin Ewert of Bundeswehr University Munich for assistance with some of the early attempts to measure flow velocity.

This material was based upon work supported by the NASA/NSF Partnership on the Science of Dusty Plasmas via NSF Award No. 1740655 and via NASA Jet Propulsion Laboratory Award No. 1573433.

\section{DATA AVAILABILITY}

The data that support the findings of this study are openly available in the CaltechDATA repository at https://doi.org/10.22002/D1. 1379.

\section{REFERENCES}

${ }^{1}$ D. D. Burgess and C. H. Skinner, "Dye laser-induced fluorescence of plasmas and its application to measurement of lower state decay-rates," J. Phys. B: At., Mol. Opt. Phys. 7(9), L297-L301 (1974).

${ }^{2}$ R. A. Stern and J. A. Johnson, "Plasma ion diagnostics using resonant fluorescence,” Phys. Rev. Lett. 34(25), 1548-1551 (1975).

${ }^{3}$ G. D. Severn, D. A. Edrich, and R. McWilliams, "Argon ion laser-induced fluorescence with diode lasers,” Rev. Sci. Instrum. 69(1), 10-15 (1998).

${ }^{4}$ A. Pikalev, M. Pustylnik, C. Räth, and H. Thomas, "Measurement of gas temperatures in dust-free and dusty argon discharges," J. Phys. D: Appl. Phys. 53(7), 075203 (2019).

${ }^{\mathbf{5}}$ R. J. Cedolin, W. A. Hargus, P. V. Storm, R. K. Hanson, and M. A. Cappelli, "Laser-induced fluorescence study of a xenon Hall thruster," Appl. Phys. B: Lasers Opt. 65(4-5), 459-469 (1997).

${ }^{6}$ V. H. Chaplin, B. A. Jorns, A. Lopez Ortega, I. G. Mikellides, R. W. Conversano, R. B. Lobbia, and R. R. Hofer, "Laser-induced fluorescence measurements of acceleration zone scaling in the $12.5 \mathrm{~kW}$ HERMeS Hall thruster," J. Appl. Phys. 124(18), 183302 (2018).

${ }^{7}$ A. K. Hansen, M. Galante, D. McCarren, S. Sears, and E. E. Scime, "Simultaneous two-dimensional laser-induced-fluorescence measurements of argon ions," in 18th Topical Conference on High-Temperature Plasma Diagnostics, Wildwood, NJ, May 16-20, 2010 [Rev. Sci. Instrum. 81(10), 10D701 (2010)].

${ }^{8}$ A. Bogaerts, R. D. Guenard, B. W. Smith, J. D. Winefordner, W. W. Harrison, and R. Gijbels, "Three-dimensional density profiles of argon metastable atoms in a direct current glow discharge: Experimental study and comparison with calculations," Spectrochim. Acta, Part B 52(2), 219-229 (1997).

${ }^{9}$ B. K. McMillin and M. R. Zachariah, "Two-dimensional laser-induced fluorescence imaging of metastable density in low-pressure radio frequency argon plasmas with added $\mathrm{O}_{2}, \mathrm{Cl}_{2}$, and $\mathrm{CF}_{4}$," J. Appl. Phys. 79(1), 77-85 (1996).

${ }^{10}$ D. S. Thompson, T. E. Steinberger, A. M. Keesee, and E. E. Scime, "Laser induced fluorescence of Ar-I metastables in the presence of a magnetic field," Plasma Sources Sci. Technol. 27(6), 065007 (2018)

${ }^{11}$ K.-B. Chai and P. M. Bellan, "Formation and alignment of elongated, fractal-like water-ice grains in extremely cold, weakly ionized plasma," Astrophys. J. 802(2), 112 (2015).

${ }^{12}$ R. S. Marshall, K.-B. Chai, and P. M. Bellan, "Identification of accretion as grain growth mechanism in astrophysically relevant water-ice dusty plasma experiment," Astrophys. J. 837(1), 56 (2017).

${ }^{13}$ T. Bieber, X. Glad, L. De Poucques, R. Hugon, J.-L. Vasseur, and J. Bougdira, "Argon ion and neutral metastable levels destruction in a magnetically enhanced inductively coupled plasma reactor," Open Plasma Phys. J. 6, 32-43 (2013).

${ }^{14}$ R. F. Kelly, K. D. Meaney, M. Gilmore, T. R. Desjardins, and Y. Zhang, “ArI/ArII laser induced fluorescence system for measurement of neutral and ion dynamics in a large scale helicon plasma," in 21st Topical Conference on High-Temperature Plasma Diagnostics, Madison, WI, Jun 05-09, 2016 [Rev. Sci. Instrum. 87(11), 11E560 (2016)].

${ }^{15}$ S. Shimizu, B. Klumov, T. Shimizu, H. Rothermel, O. Havnes, H. M. Thomas, and G. E. Morfill, "Synthesis of water ice particles in a plasma chamber," J. Geophys. Res. 115, D18205, https://doi.org/10.1029/2009jd013375 (2010).

${ }^{16}$ K.-B. Chai and P. M. Bellan, "Spontaneous formation of nonspherical water ice grains in a plasma environment," Geophys. Res. Lett. 40(23), 6258-6263, https://doi.org/10.1002/2013gl058268 (2013).

${ }^{17}$ M. J. Goeckner, J. Goree, and T. E. Sheridan, "Saturation broadening of laserinduced fluorescence from plasma ions," Rev. Sci. Instrum. 64(4), 996-1000 (1993).

${ }^{18}$ M. Y. Pustylnik, M. A. Fink, V. Nosenko, T. Antonova, T. Hagl, H. M. Thomas, A. V. Zobnin, A. M. Lipaev, A. D. Usachev, V. I. Molotkov, O. F. 
Petrov, V. E. Fortov, C. Rau, C. Deysenroth, S. Albrecht, M. Kretschmer, M. H. Thoma, G. E. Morfill, R. Seurig, A. Stettner, V. A. Alyamovskaya, A. Orr, E. Kufner, E. G. Lavrenko, G. I. Padalka, E. O. Serova, A. M. Samokutyayev, and S. Christoforetti, "Plasmakristall-4: New complex (dusty) plasma laboratory on board the International Space Station," Rev. Sci. Instrum. 87(9), 093505 (2016).

${ }^{19}$ A. K. Chernyshov, P. A. Mikheyev, N. N. Lunev, and V. N. Azyazov, "Pressure shift coefficient measurements in an RF discharge for Ar 45[3/2](2)-5p[3/2](3) transition with the help of diode-laser absorption spectroscopy," in 26th Annual
International Laser Physics Workshop (LPHYS'17), volume 999 of Journal of Physics Conference Series, 2018 (Kazan E K Zavoisky Phys Tech Inst, Kazan, Russia, 2017)

${ }^{20}$ M. Aramaki, K. Ogiwara, S. Etoh, S. Yoshimura, and M. Y. Tanaka, "High resolution laser induced fluorescence Doppler velocimetry utilizing saturated absorption spectroscopy," Rev. Sci. Instrum. 80(5), 053505 (2009).

${ }^{21}$ A. Bondi, "Van der Waals volumes + radii," J. Phys. Chem. 68(3), 441 (1964).

${ }^{22}$ P. Pyykkö, "Strong closed-shell interactions in inorganic chemistry," Chem. Rev. 97(3), 597-636 (1997). 